\title{
Generating Self-Contained and Summary-Centric Question Answer Pairs via Differentiable Reward Imitation Learning
}

\author{
Li Zhou Kevin Small Yong Zhang Sandeep Atluri \\ Amazon Alexa \\ \{lizhouml,smakevin,yonzhn,satluri\}@amazon.com
}

\begin{abstract}
Motivated by suggested question generation in conversational news recommendation systems, we propose a model for generating question-answer pairs (QA pairs) with selfcontained, summary-centric questions and length-constrained, article-summarizing answers. We begin by collecting a new dataset of news articles with questions as titles and pairing them with summaries of varying length. This dataset is used to learn a QA pair generation model producing summaries as answers that balance brevity with sufficiency jointly with their corresponding questions. We then reinforce the QA pair generation process with a differentiable reward function to mitigate exposure bias, a common problem in natural language generation. Both automatic metrics and human evaluation demonstrate these QA pairs successfully capture the central gists of the articles and achieve high answer accuracy. ${ }^{1}$
\end{abstract}

\section{Introduction}

Automatic generation of question-answer pairs (QA pairs) is a widely studied problem, primarily used to improve the performance of question answering systems via data augmentation (Alberti et al., 2019; Shakeri et al., 2020). However, question generation has also recently garnered interest in the context of conversational agents, where suggested questions (SQs) (i.e., You can also ask...) have emerged as a promising approach to drive multi-turn dialogues by educating customers about the agent capabilities and guiding users along dialogue trajectories with more engaging content (Yin et al., 2020; Nouri et al., 2020).

As an example, consider a news chatbot engaged in a dialogue regarding COVID-19 vaccine developments producing the SQ \{Q: How effective is the Pfizer-BioNTech vaccine?\} paired with the answer

\footnotetext{
${ }^{1}$ Code and Data will be made available at https:// github.com/amazon-research/SC2QA-DRIL
}

\{A: Pfizer/BioNTech vaccine is around $91 \%$ effective at preventing COVID-19, according to updated trial data. Experts fear new variants of COVID-19 from South Africa and Brazil may be resistant to existing vaccines and treatment. $\}$ Firstly, SQs of this form mitigates the user burden regarding the necessity of both deep subject knowledge to ask good questions and awareness of the agent question answering capabilities to expect good answers. Secondly, the agent can look-ahead when selecting SQs to bias toward confidently correct answers and content expected to lead to further follow-up questions and general system engagement.

Targeting the SQ problem in news chatbot scenarios (e.g., (Laban et al., 2020)), this work examines QA pair generation corresponding to a news article summary paired with a self-contained question. Table 1 shows an example of the task. SQs based on these summary-centric QA pairs act as implicit article recommendations, complementing SQs focusing on passage-level extracted answers or factoid information. QA pairs generated for this purpose must satisfy several criteria including: (1) questions are self-contained (i.e., users need not read the corresponding articles nor require significant additional domain knowledge to unambiguously understand the questions (Yin et al., 2020)), (2) questions are summary-centric (questions capture the gists of the corresponding articles), (3) answers correctly answer the questions, and (4) answers are brief but sufficient such that users can confidently trust the results. Additionally, to support different settings (e.g., screened device, mobile device, voice-only), we explore QA pair generation for varying application-specific answer length requirements.

To satisfy these requirements, we first collect a corpus of suitable QA pairs, accomplished by curating a set of news articles with well-formed questions as their titles and for which we can confidently generate variable length summaries as an- 


\begin{tabular}{l} 
Article: President Biden's infrastructure plan calls \\
for an unprecedented boost in federal aid to the na- \\
tion's passenger rail system, seeking to address Am- \\
trak's repair backlog, extend service to more cities \\
and modernize the network in the Northeast Corri- \\
dor. The American Jobs Plan announced Wednes- \\
day calls for \$80 billion for rail - money that could \\
be crucial in taking passenger service to cities such \\
as Las Vegas and Nashville, and expand operations \\
across large metropolitan areas such as Atlanta \\
and Houston. "President Biden's infrastructure \\
plan is what this nation has been waiting for," Am- \\
trak chief executive William J. Flynn said, while \\
echoing Biden's push to rebuild and improve... \\
\hline Suggested Question: What does President Biden's \\
infrastructure plan mean for Amtrak? \\
Short Answer: The federal funding would help \\
Amtrak accomplish long-needed upgrades to tracks, \\
tunnels and bridges in the Northeast. \\
Long Answer: The American Jobs Plan an- \\
nounced Wednesday calls for \$80 billion for rail. \\
The federal funding would help Amtrak accom- \\
plish long-needed upgrades to tracks, tunnels and \\
bridges in the Northeast, the nations busiest rail \\
corridor. Amtrak has a \$45.2 billion backlog of \\
projects that it says are needed to bring its assets \\
to a state of good repair. \\
\end{tabular}

Table 1: The suggested QA pair generation task. Given an article, we generate a self-contained and summarycentric question and a length-constrained answer. The question captures the gist of the article and can be understood without reading the corresponding article.

swers. Observing that the summary generation $\rightarrow$ question generation pipeline suffers from exposure bias (Ranzato et al., 2016), we propose a novel differential reward imitation learning (DRIL) training method that samples summary answers and reconstructs questions exclusively based on the hidden states of the answer decoder. Generated summaries are capable of directly reconstructing the questions, making them more likely the answers to the questions, and generate questions more closely related to the gists of the articles. We empirically validate the model with automated and human evaluations.

In this paper, we study QA pair generation corresponding to variable length article-summarizing answers paired with self-contained and summarycentric questions. Our contributions include: (1) We collect a new QA dataset targeted for producing SQs in a news chatbot. (2) We propose a QA pair generation model where both questions and answers are well-formed, questions capture the central gists of articles, and answers are succinct while containing sufficient supporting context. (3) We propose a novel differentiable reward imita- tion learning (DRIL) method which shows better performance over maximum likelihood estimation (MLE) and reinforcement learning (RL) for QA pair generation. (4) We perform extensive empirical evaluations to quantify DRIL-based QA pair generation improvements.

\section{Related Works}

Question-only Generation (QG). Both heristicbased (Heilman and Smith, 2010) and neural models (Du et al., 2017; Zhou et al., 2017; Sun et al., 2018) have been applied to QG. Usually, neural QG models are given contexts containing answers beforehand, contrasting with our goal of jointly generating QA pairs. Tuan et al. (2020); Song et al. (2018); Zhao et al. (2018) proposed to generate questions from long text and wider contexts, which is related to our method for QG using summaries. However, these wider contexts are only used to improve QG for the specified answer spans and do not attempt to capture the central gists of articles.

Question and Answer Generation (QG+AG). QG+AG generates QA pairs jointly (Liu et al., 2020; Alberti et al., 2019; Du and Cardie, 2018, 2017; Subramanian et al., 2018; Wang et al., 2019; Krishna and Iyyer, 2019), frequently with two independent steps: identify question-worthy answer spans followed by generating answer-aware questions. Recent works train neural models to generate QA pairs (Shakeri et al., 2020; Lee et al., 2020) using QA datasets such as SQuAD (Rajpurkar et al., 2016) and Natural Questions (Kwiatkowski et al., 2019) modulo the goal of generating self-contained questions paired with succinct but sufficient articlesummarizing answers.

Applications of QG and QG+AG. QG and QG+AG have been used for applications including data augmentation for QA systems (Alberti et al., 2019; Shakeri et al., 2020), information seeking in chatbots (Qi et al., 2020a; Laban et al., 2020), document understanding (Krishna and Iyyer, 2019), educational practice and assessment (Le et al., 2014), and online shopping (Yu et al., 2020).

Training Mechanism for Sequence Prediction. Sequence prediction models are commonly trained with MLE. However, MLE can lead to degeneration (Holtzman et al., 2019) caused by exposure bias (Ranzato et al., 2016). Many algorithms (Yu et al., 2017; Lamb et al., 2016; Song et al., 2020; Welleck et al., 2019) have been proposed to mitigate exposure bias. Our DRIL method not only 
mitigates exposure bias, but also optimizes for a differentiable reward function that is aligned with the end goal. Please refer to Section 4.2 for comparison between DRIL and existing algorithms.

\section{$3(\mathcal{S C})^{2} \mathcal{Q} \mathcal{A}$ : A Self-Contained and Summary-Centric QA Dataset}

While multiple QA datasets exist to train a QG or AG model, none specifically fit the goal of this paper. QA pairs in SQuAD (Rajpurkar et al., 2018), NewsQA (Trischler et al., 2017), and Natural Questions (NQ) (Kwiatkowski et al., 2019) are not designed to capture the article gists, and a significant number of questions in SQuAD and NewsQA are not self-contained.

A key observation enabling this work is that many news articles have questions as their titles (e.g. How has the Biden administration helped student loan borrowers?) that can be used to train a SQ generation model since these questions usually correspond to the central gists of the news articles and are designed to be understood without reading the articles. However, two challenges remain: (1) clickbait titles need to be filtered, and 2) these questions are not paired with summary-centric answers. Therefore, we developed the following data collection procedure to produce $(\mathcal{S C})^{2} \mathcal{Q A}$, our selfcontained summary-centric QA dataset.

\subsection{Question-Article Pairs Collection}

Starting with a curated URL list of news websites, we collected all articles between September 2020 to March 2021 with a title that starts with a predefined list of words (e.g., Where, What, How) and ends with a question mark. We then define a set of rules to filter out ill-formed and clickbait titles (details in Appendix A). Finally, we remove any questions that appear in the articles to ensure we don't learn to copy the questions when present In total, we collected 39,460 such question-article pairs.

\subsection{Question, Article, Summary, Length Constraint\} 4-Tuples Collection}

Given collected question-article pairs, we must pair them with suitable answers to produce QA pairs. From a preliminary study, we observed that $\sim 70 \%$ of title questions can be answered by summaries of the corresponding articles. As a result, we set out to augment the question-article dataset with generated summaries as pseudo ground truth answers using following three-step procedure:

Step 1 (Define desired answer lengths): One of our goals is to generate well-formed answers that are succinct while containing sufficient supporting context. Therefore, we generate summaries with varying brevity. Analyzing the average number of tokens for the first 1,2 and 3 sentences of the CNN/DailyMail summaries (Hermann et al., 2015), we define three buckets of varying answer lengths: $(0,30],(30,50]$ and $(50,72]$ BPE tokens.

Step 2 (Generate summary): For each article and desired length bucket, we use three SoTA summarization models (PEGASUS (Zhang et al., 2020), BART (Lewis et al., 2020), and CTRLSum (He et al., 2020)) fine-tuned on CNN/DailyMail to generate three candidate summaries - enforcing summary length via control of EOS token generation. Unfinished sentences are removed and the length bucket is reassigned if needed.

Step 3 (Filter-out incorrect summary answers): Not all questions can be answered by the generated summaries since: (1) even the ground truth summary may not be a correct answer to the question and (2) summaries generated by SoTA models may not be good. To identify if a candidate summary answers the question, we train a QA pair classifier using the 4 million question-snippet pairs MSMARCO dataset (Bajaj et al., 2016). For each article and length bucket, we select the candidate summary that has the highest score predicted by the trained classifier. In total, we produce 53,746 4-Tuples of \{Question, Article, Summary, Length Constraint . For additional details and dataset statistics, please refer to Appendix A.

\section{Models for QA Pair Generation}

In this section, we propose a family of QA pair generation models that are trained on the data collected in Section 3. Let $D$ denote a document (news article), $S$ denote a summary, $Q$ denote a question, $L$ denote a length bucket indicator ( $\mathrm{LB} 0, \mathrm{LB} 1$ or LB2), and $\langle$ s $>$ and $\langle/ s\rangle$ denote the special BOS and SEP tokens respectively.

\subsection{Base $\mathrm{D} \rightarrow \mathrm{S} \rightarrow \mathbf{Q}$ Model (D-S)}

Our base model is shown in Figure 1, consisting of two transformer-based encoder-decoder models (Vaswani et al., 2017) where one performs answer generation (AG) and the other question generation $(\mathrm{QG})$. During training, the AG model encodes a concatenation of the length bucket indicator and 


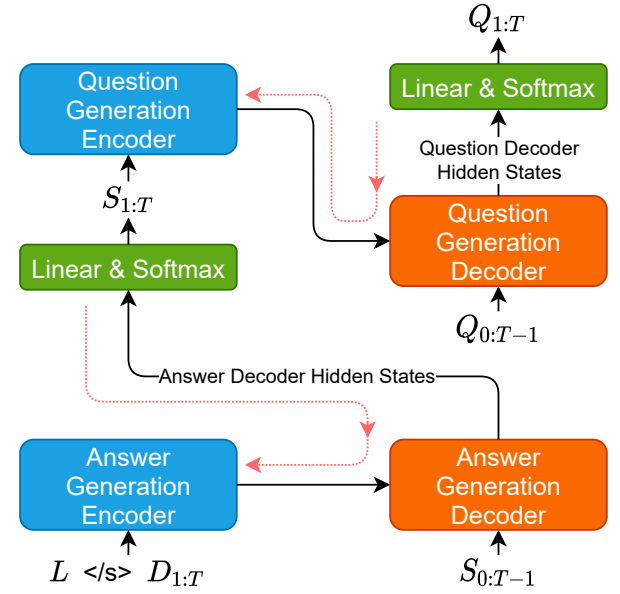

Figure 1: Training of answer generation (AG) and question generation (QG) of the D-S model. L, D, S, Q denotes the length bucket indicator, document, summary, and question, respectively. Red dash arrows denote gradient flow.

the document, and decodes a length-constrained summary:

$$
\begin{aligned}
& f_{\theta_{\mathrm{enc}}^{a}}: L+D \rightarrow c_{\mathrm{enc}}^{a} \\
& f_{\theta_{\mathrm{dec}}^{a}}: S_{0: T-1}, c_{\mathrm{enc}}^{a} \rightarrow S_{1: T}
\end{aligned}
$$

where $\theta_{\mathrm{enc}}^{a}$ and $\theta_{\mathrm{dec}}^{a}$ are the encoder and decoder parameters, $c_{\mathrm{enc}}^{a}$ is the sequence of hidden states at the last encoder layer, $S_{1: T}$ is the ground truth summary, and $S_{0: T-1}$ is the decoder input $\left(S_{1: T}\right.$ offset by one timestamp and prepended by a BOS token). The AG model is trained using MLE:

$$
\mathcal{L}\left(\theta_{\mathrm{enc}}^{a}, \theta_{\mathrm{dec}}^{a}\right)=-\sum_{n=1}^{N} \log p\left(S^{(n)} \mid L^{(n)}+D^{(n)}\right)
$$

where $(n)$ represents the $n$-th training instance. QG is also trained via MLE, mapping an input summary to a question:

$$
\begin{gathered}
f_{\theta_{\mathrm{enc}}^{q}}: S \rightarrow c_{\mathrm{enc}}^{q} \\
f_{\theta_{\mathrm{dec}}^{q}}: Q_{0: T-1}, c_{\mathrm{enc}}^{q} \rightarrow Q_{1: T} \\
\mathcal{L}\left(\theta_{\mathrm{enc}}^{q}, \theta_{\mathrm{dec}}^{q}\right)=-\sum_{n=1}^{N} \log p\left(Q^{(n)} \mid S^{(n)}\right)
\end{gathered}
$$

During inference, when decoding summary answers, we again control the generation of EOS to fall into the range specified by the desired length bucket. We remove any unfinished sentences at the end unless after the truncation the answer is shorter than the minimum length of the length bucket.

We use a pre-trained BART model (Lewis et al., 2020) to initialize $\theta_{\mathrm{enc}}^{a}, \theta_{\mathrm{dec}}^{a}, \theta_{\mathrm{enc}}^{q}$ and $\theta_{\mathrm{dec}}^{q}$. We name this base model D-S since the AG model takes the document (D) as input and the QG model takes the summary (S) as input. In Section 4.3 we will describe multiple variants of this model.

\subsection{Optimizing Answer Generation by Differentiable Rewards}

When using MLE to train the base model, the decoder input at timestep $t$ is the ground truth token at timestep $t-1$, sometimes called teacherforcing (Williams and Zipser, 1989) and known to suffer from exposure bias (Ranzato et al., 2016) due to the mismatch between training and inference. That is, during inference the decoder input is the predicted token instead of the ground truth token of the last timestep, causing errors from each timestamp to accumulate during generation. It has been shown that neural text generation models trained with MLE lead to generic and repetitive outputs (Welleck et al., 2019; Holtzman et al., 2019). Additionally, we usually want to optimize generation metrics (e.g., ROUGE) and human feedback directly instead of optimizing training data likelihood. To mitigate these concerns, we can sample decoder output during training and calculate the loss of the sampled output. Several works use RL to achieve this for text generation (Stiennon et al., 2020; Ziegler et al., 2019; Yu et al., 2017) and directly optimize for preferred metrics. However, $\mathrm{RL}$ is not sample efficient and difficult to tune in text generation tasks due to sparse rewards. For example, Hosking and Riedel (2019) have shown that applying RL to QG do not improve human evaluation metrics.

Meanwhile, we observe that when generating a summary as the answer of a QA pair, we want to generate a summary that can better reconstruct the ground truth question without the article since: (1) a summary that can reconstruct a question is more likely to be able to answer that question and (2) a summary that better reconstructs the ground truth question leads to a generated question that is closer to the gist of the article. Moreover, the AG model is conditioned on the length bucket to control the levels of brevity, meaning that when the maximum allowed answer length is short, the question reconstruction will enforces the AG model to generate succinct but informative answers with respect to the question given the selected brevity level. We validate these assumptions in Section 5.

We now propose the differentiable reward imita- 


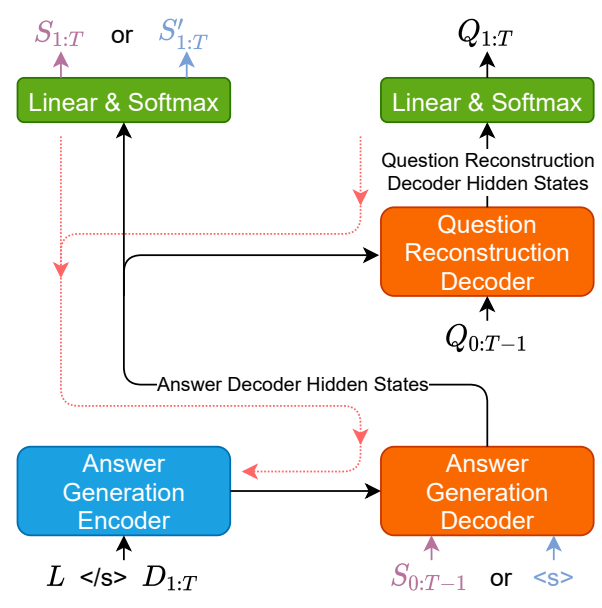

Figure 2: Training of answer generation (AG) of the D-S-DRIL model. The input to the AG decoder is either $S_{0: T-1}$ or $\langle\mathrm{S}\rangle$. When the input is $S_{0: T-1}$, the AG decoder uses teacher-forcing to predict $S_{1: T}$, and the gradients back-propagate from $S_{1: T}$ to the AG decoder and AG encoder (the red dash arrow on the middle left), which is similar to the AG of the D-S model. However, when the input is $\langle\mathrm{S}\rangle$, the AG decoder samples a summary $S_{1: T}^{\prime}$, and the answer decoder hidden states are used to reconstruct the question $Q_{1: T}$. The gradients back-propagate from $Q_{1: T}$ to the AG decoder and $\mathrm{AG}$ encoder (the red dash arrow on the top right). This reinforces the model to generate summaries that can reconstruct the questions.

tion learning (DRIL) method for training the AG model as shown in Figure 2. During training, the AG model performs vanilla sampling to generate a summary:

$$
\begin{aligned}
& f_{\theta_{\mathrm{enc}}^{a}}: L+D \rightarrow c_{\mathrm{enc}}^{a} \\
& f_{\theta_{\mathrm{dec}}^{a}}: \mathrm{BOS}, c_{\mathrm{enc}}^{a} \rightarrow c_{\mathrm{dec}}^{a}, S^{\prime}
\end{aligned}
$$

where $c_{\mathrm{dec}}^{a}$ is the sequence of hidden states at the last layer of the decoder, and $S^{\prime}$ is the sampled summary. This differs from teacher-forcing since summaries are sampled in training. We then use another transformer-based decoder to reconstruct the question:

$$
f_{\theta_{\mathrm{dec}}^{r}}: Q_{0: T-1}, c_{\mathrm{dec}}^{a} \rightarrow Q_{1: T}
$$

noting that this decoder only depends on the hidden states of the AG decoder (not $L+D$ ). This forces the model to reconstruct the question only from the summary. The gradient can back-propagate from the question to the hidden states of the AG decoder $c_{\mathrm{dec}}^{a}$ and $\mathrm{AG}$ encoder $c_{\mathrm{enc}}^{a}$ such that the question reconstruction loss will guide AG. To ensure generated summary fluency, we also add the MLE loss from the base model. Overall, the AG model's loss function is given by:

$$
\begin{aligned}
& \mathcal{L}\left(\theta_{\mathrm{enc}}^{a}, \theta_{\mathrm{dec}}^{a}, \theta_{\mathrm{dec}}^{r}\right)= \\
& \quad-\sum_{n=1}^{N} \lambda \log p\left(Q^{(n)} \mid S^{(n)}, L^{(n)}+D^{(n)}\right) \\
& \quad+(1-\lambda) \log p\left(S^{(n)} \mid L^{(n)}+D^{(n)}\right)
\end{aligned}
$$

In our experiments, $\lambda=0.3$ performs the best on the validation set. Finally, while we apply DRIL to the training of the AG model, the QG model remains the same as the base model. We do not use the question reconstruction decoder $\theta_{d e c}^{r}$ as our QG model because its encoder input $c_{d e c}^{a}$ is a unidirectional representation and hence not preferred. We call this QA pair generation model D-S-DRIL.

Connection with RL, Unlikelihood (Welleck et al., 2019), SeqGAN (Yu et al., 2017), and Professor-forcing (Lamb et al., 2016), etc. These methods mitigate exposure bias to some degree by calculating the loss from sampled sequences during training. Unlikelihood training penalizes the likelihood of undesired sampled sequences. SeqGAN and Professor-forcing both calculate the loss using a discriminator which learns to distinguish between the generated and ground truth sequences. They don't optimize an extrinsic reward function. Caccia et al. (2019) show that Language GANs suffer from mode collapse and do not outperform MLE on the quality and diversity evaluation. SeqGAN uses RL optmization and thus suffers from aforementioned issues. Our DRIL method, on the other hand, learns to optimize a differentiable reward function that aligns with the end goal, and has lower gradient variance compared with RL. We empirically compare RL with DRIL in Section 5.

Beyond this work, DRIL can be applied to other sequence prediction problems. For example, in step-by-step instruction following such as ALFRED tasks (Shridhar et al., 2020), DRIL can optimize the current step's action trajectory such that it can reconstruct the next $K$ instructions. The intuition is if the current step's action trajectory is correct, then the agent should be able to follow the ground truth actions in the next steps to fulfill the tasks. From this perspective, DRIL is similar to SQIL (Reddy et al., 2020), which avoids drifting away from the demonstrations over long horizons by encouraging trajectories that return to demonstrated states when encountering unseen states. In conversational AI, Hosseini-Asl et al. 
(2020) proposed to fine-tune a GPT-2 model to generate system responses turn-by-turn. DRIL can optimize response generation at each turn such that the response and dialogue context can reconstruct the next $K$ turns' user and system response with a similar intuition: a correct system response will increase the likelihood of the ground truth in future turns. It avoids drifting away from demonstrations and mitigates exposure bias.

\subsection{Base Model Variants}

In this section, we specify additional baseline QA pair generation models. Similar to the base D$\mathrm{S}$ model, these models are based on transformer encoder-decoder architectures. The differences between these models are the encoder and decoder inputs during training and inference as summarized in Table 2. Models are named by the encoder input of the AG and QG models joined with a '-'. D-D is similar to D-S except that QG takes the document (D) rather than the summary (S) as encoder input. QD-D generates question-conditioned answers, such that the AG model becomes a questionanswering model. D-SD is an extension of D-S and D-D such that the encoder of the QG model takes the concatenation of S and D. D-S-DRIL optimizes the AG model of D-S using DRIL. D-S-RL optimizes the AG model of D-S using RL, and the reward function is defined as the negative question reconstruction loss calculated by the QG model of D-S. For further details, refer to Appendix B.

\section{Experiments}

We conduct experiments to answer 3 research questions: (1) How good are the QA pairs generated by each algorithm?, (2) Can DRIL outperform MLE and $R L$ on QA pair generation?, and (3) Is our $(\mathcal{S C})^{2} \mathcal{Q A}$ dataset preferable compared with existing public QA datasets for QA pair generation? For each generated QA pair, we are interested in evaluating the following 3 questions: (1) Does the length-constrained summary answer the question?, (2) Does the question capture the article gist?, (3) Is the question self-contained? We specify automated metrics and human evaluations to quantify the answers to these research questions.

\subsection{Automated Metrics}

ROUGE-L (R-L) and BLEU. ROUGE-L and BLEU evaluate generated summaries/questions with respect to reference summaries/questions in the validation set.

QA Pair Classifier Scores (QACS). We need to measure how well the generated summaries answer the generated questions despite not having ground truth answers. Using the trained QA pair classifier from Section 3, we propose QACS, which is the average of classifier predicted scores on the generated QA pairs. The pseudo upper and lower bounds of QACS are 0.359 and 0.046 based on the average classifier predicted scores of the positive and negative QA pairs in our human evaluation.

\subsection{Human Evaluation}

We conduct human evaluation on Amazon Mechanical Turk. We designed 7 annotation tasks (ATs). Please refer to Appendix $\mathrm{C}$ for detailed human evaluation setup. Here we describe 4 ATs for which we are most concerned: AT-1 shows a QA pair and asks Without referring to the answer or the article, are you able to understand the question? (Is the question self-contained?), AT-2 follows AT-1 and asks Does the passage in the Answer text box answers the question?, AT-5 shows the corresponding article and asks Does the question in the Question text box capture the gist of the Article?. For these three tasks, annotators select either TRUE or FALSE. AT-6 shows an article and a list of questions generated by different models and asks Which Question shown above best captures the gist of the Article?

\subsection{Baseline}

We evaluate D-S and its variants in Table 2. Beyond that, we evaluate the following baselines. QAGen 2S: This is the state-of-the-art model for QA pairs generation for improving QA systems. We train QAGen 2S on our dataset, which is similar to QD-D except that there is no length control on the answers. CTRLSum: We use a pretrained CTRLSum model to generate questiondependent summaries. Questions are generated by the QG model of QD-D. QA Transfer: We train a question-answering model on the NewsQA dataset to answer the generated questions. Questions are generated by the QG model of QD-D. This is to verify if a pre-trained question-answering model is sufficient to answer the questions in our dataset. D-S-NewsQA and Natural Questions (D-S-NQ): These two models are similar to D-S, except that the QG models are trained on NewsQA and NQ, respectively. This is to verify if $(\mathcal{S C})^{2} \mathcal{Q A}$ is better than other existing QA datasets for QG tasks. Refer to Appendix B for implementation details. 


\begin{tabular}{|c|c|c|c|c|c|c|c|c|}
\hline & \multicolumn{4}{|c|}{ Training } & \multicolumn{4}{|c|}{ Inference } \\
\hline & \multicolumn{2}{|c|}{ Answer Generation } & \multicolumn{2}{|c|}{ Question Generation } & \multicolumn{2}{|c|}{ Answer Generation } & \multicolumn{2}{|c|}{ Question Generation } \\
\hline & Encoder & Decoder & Encoder & Decoder & Encoder & Decoder & Encoder & Decoder \\
\hline D-S & $\mathrm{L}+\mathrm{D}$ & $\mathrm{S}$ & $\mathrm{S}$ & Q & $\mathrm{L}+\mathrm{D}$ & $S^{\prime}$ & $S^{\prime}$ & $Q^{\prime}$ \\
\hline D-D & $\mathrm{L}+\mathrm{D}$ & $\mathrm{S}$ & $\mathrm{D}$ & Q & $\mathrm{L}+\mathrm{D}$ & $\mathrm{S}^{\prime}$ & $\mathrm{S}^{\prime}$ & $Q^{\prime}$ \\
\hline D-SD & $\mathrm{L}+\mathrm{D}$ & $S$ & $\mathrm{~S}+\mathrm{D}$ & $\mathrm{Q}$ & $\mathrm{L}+\mathrm{D}$ & $\mathrm{S}^{\prime}$ & $S^{\prime}+D$ & $\mathrm{Q}^{\prime}$ \\
\hline QD-D & $\mathrm{Q}+\mathrm{L}+\mathrm{D}$ & $\mathrm{S}$ & $\mathrm{D}$ & Q & $Q^{\prime}+\mathrm{L}+\mathrm{D}$ & $S^{\prime}$ & $\mathrm{D}$ & $Q^{\prime}$ \\
\hline D-S-DRIL & $\mathrm{L}+\mathrm{D}$ & $\mathrm{S} / \mathrm{S}^{\prime}$ & $\mathrm{S}$ & Q & $\mathrm{L}+\mathrm{D}$ & $S^{\prime}$ & $\mathrm{S}^{\prime}$ & $Q^{\prime}$ \\
\hline D-S-RL & $\mathrm{L}+\mathrm{D}$ & S/S & $\mathrm{S}$ & Q & $\mathrm{L}+\mathrm{D}$ & $\mathrm{S}^{\prime}$ & $S^{\prime}$ & $Q^{\prime}$ \\
\hline QAGen 2S & $\mathrm{D}+\mathrm{Q}$ & $\mathrm{S}$ & $\mathrm{D}$ & Q & $\mathrm{D}+\mathrm{Q}^{\prime}$ & $S^{\prime}$ & $\mathrm{D}$ & $Q^{\prime}$ \\
\hline
\end{tabular}

Table 2: A summary of models (D-S and its variants) we proposed for QA pair generation. Q' and S' denote the question and answer generated during inference, respectively. QAGen 2S (Shakeri et al., 2020) is a state-of-the-art baseline. A full table that includes all the baselines in our experiments is shown at Appendix Table 15.

\subsection{Data}

Training and Validation set. We use the data described in Section 3 for training, taking the last 5,000 out of the 53,746 examples as validation set. Test set. It is desirable to evaluate models on articles that do not use questions as titles. We sampled news articles between April 1 to April 7, 2021 from the following news domains: washingtonpost.com, reuters.com, foxnews.com, cnn.com, cbsnews.com, nbcnews.com, nydailynews.com. We filtered out articles that use questions as titles, and removed all questions in the articles. In total we collect 7,698 test examples. Unlike validation set, there are no ground truth questions or answers in the test set.

\subsection{Quality of Generated Answers}

In this section we measure the quality of answers, particularly, whether they answer the corresponding questions. In Table 3, we show the ROUGE-L score of predicted summaries on the validation set, and QACS and AT-2 accuracy on the test set, resulting in the following observation.

Models that generate questions based on answers have higher QACS and AT-2 accuracy than models that generate answers based on questions. Recall that during inference, D-S, D-D, D-S-DRIL and D-S-RL first generate summaries as answers and then generate questions based on the answers (see Table 2). These algorithms perform much better than QD-D, CTRLSum, QAGen 2S and QA Transfer which first generate questions and then generate answers to these questions. For example, D-S achieves $51.2 \%, 39.6 \%$, and $23.4 \%$ higher AT-2 accuracy than QAGen $2 \mathrm{~S}$ in each of the 3 length buckets respectively. This observation is consistent in both QACS and AT-2 accuracy. Meanwhile, QD-D achieves the best ROUGE-L scores while the QACS and AT-2 accuracy are significantly lower than D-S (e.g., AT-2 accuracy is
$33.9 \%$ lower than D-S in length bucket 0). All these observations show that, to ensure the generated questions and answers match with each other, we should generate questions from answers rather than the opposite direction. This is especially true on our dataset, because the ground truth answers of our dataset are summaries, which are generated without conditioning on the questions (modulo examples generated by the CTRLSum in Section 3).

\subsection{Quality of Generated Questions}

\subsubsection{Results on $(\mathcal{S C})^{2} \mathcal{Q A}$ Dataset}

In this section, we evaluate the quality of generated questions, particularly, whether the questions capture the gists of articles. From Section 5.5 we already observed that only D-D, D-S, D-S-DRIL, and D-S-RL can generate high quality answers. Therefore, here we only focus on these four models (refer to Appendix C and Section 5.7 for results on other models). The results are shown in Table 4 . We report ROUGE-L/BLEU score of predicted questions on the validation set. Questions are predicted from predicted summaries instead of ground truth summaries, which is consistent with inference on the test set where we also don't have ground truth summaries. We also report AT-5 accuracy on test set and make the following observations.

DRIL and RL reinforce AG with question reconstruction loss and thus better reconstruct ground truth questions on validation set and better capture gists of articles on test set. Table 4 shows that D-S-DRIL achieves higher ROUGE$\mathrm{L}$ and BLEU score than D-S across all the length buckets. Note that D-S and D-S-DRIL have the same QG model so the only difference is the AG model, showing that D-S-DRIL is able to generate better summaries that can better reconstruct the ground truth questions. This aligns with our goal of designing the question reconstruction loss. Mean- 


\begin{tabular}{|c|c|c|c|c|c|c|c|c|c|}
\hline & \multicolumn{3}{|c|}{ length bucket 0} & \multicolumn{3}{|c|}{ length bucket 1} & \multicolumn{3}{|c|}{ length bucket 2} \\
\hline & R-L & QACS & AT-2 Accuracy & R-L & QACS & AT-2 Accuracy & R-L & QACS & AT-2 Accuracy \\
\hline D-S & \multirow{3}{*}{59.993} & 0.219 & $0.623 \pm 0.052$ & \multirow{3}{*}{54.110} & 0.255 & $0.748 \pm 0.045$ & \multirow{3}{*}{52.236} & 0.294 & $0.754 \pm 0.046$ \\
\hline D-D & & 0.176 & $0.571 \pm 0.053$ & & 0.224 & $0.683 \pm 0.048$ & & 0.268 & $0.782 \pm 0.045$ \\
\hline D-SD & & 0.125 & $0.403 \pm 0.072$ & & 0.184 & $0.547 \pm 0.074$ & & 0.235 & $0.653 \pm 0.071$ \\
\hline QD-D & 62.219 & 0.110 & $0.412 \pm 0.072$ & 55.200 & 0.167 & $0.508 \pm 0.071$ & 53.075 & 0.212 & $0.574 \pm 0.072$ \\
\hline D-S-DRIL & $\overline{58.153}$ & 0.225 & $0.631 \pm 0.049$ & $\overline{53.376}$ & 0.263 & $0.771 \pm 0.042$ & $\overline{50.816}$ & 0.304 & $0.814 \pm 0.038$ \\
\hline D-S-RL & 59.466 & 0.224 & 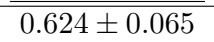 & 53.635 & 0.262 & $0.733 \pm 0.060$ & 51.871 & 0.302 & $\overline{0.813 \pm 0.053}$ \\
\hline CTRLSum & 48.973 & 0.040 & $0.112 \pm 0.046$ & 52.766 & 0.132 & $0.438 \pm 0.073$ & 50.205 & 0.183 & $0.530 \pm 0.075$ \\
\hline QAGen 2S & 56.881 & 0.112 & $0.412 \pm 0.073$ & 52.912 & 0.171 & $0.536 \pm 0.072$ & 51.741 & 0.218 & $0.611 \pm 0.071$ \\
\hline QA Transfer & - & 0.091 & $0.521 \pm 0.071$ & - & 0.128 & $0.587 \pm 0.070$ & - & 0.156 & $0.687 \pm 0.065$ \\
\hline
\end{tabular}

Table 3: Evaluation of Answer Quality. Underline, bold, and bold represent the best results on ROUGE-L (R-L),

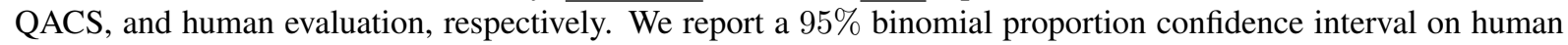
evaluation. D-S-DRIL generates higher quality answers than baselines in all three answer length bucket on test set.

while, we assume that in our dataset the ground truth questions capture the gists of articles, this means that, by optimizing question reconstruction loss, D-S-DRIL can generate questions that better capture the gists of articles. This is validated by the results on AT-5 accuracy. D-S-DRIL has about $6 \%$ and $3 \%$ higher AT-5 accuracy than D-S on length bucket 0 and 1 , respectively. D-S-DRIL has lower AT-5 accuracy than D-S on length bucket 2 , likely because when the maximum allowed summary length is long, there is sufficient information to reconstruct the questions even without the reconstruction loss. D-S-DRIL also shows better performance compared with D-S-RL, indicating the advantage of differentiable question reconstruction loss over the non-differentiable question reconstruction reward.

AT-6 shows one article and a list of questions generated by D-D, D-S, D-S-DRIL, and D-S-RL. Annotators select the question that best captures the gist of the displayed article. Figure 3 shows the percentage of each model selected. We can see that questions generated by D-S-DRIL are preferred in length bucket 0 and 1 , which is consistent with our results in Table 4.

\subsection{2 $(\mathcal{S C})^{2} \mathcal{Q A}$ v.s. Exiting QA Datasets}

In this section, we evaluate if $(\mathcal{S C})^{2} \mathcal{Q A}$ is better than existing publicly available QA datasets for QG. We compare with D-S-NewsQA and DS-NQ. NewsQA and NQ datasets are designed for question-answering but not QG specifically. Similar to $(\mathcal{S C})^{2} \mathcal{Q A}$, NewsQA is in news domain but without explicitly self-contained questions. For example, the question "what are they going to address?" in the NewsQA dataset is incomprehensible without reading the article due to lack of pronoun resolution. The human evaluation results are

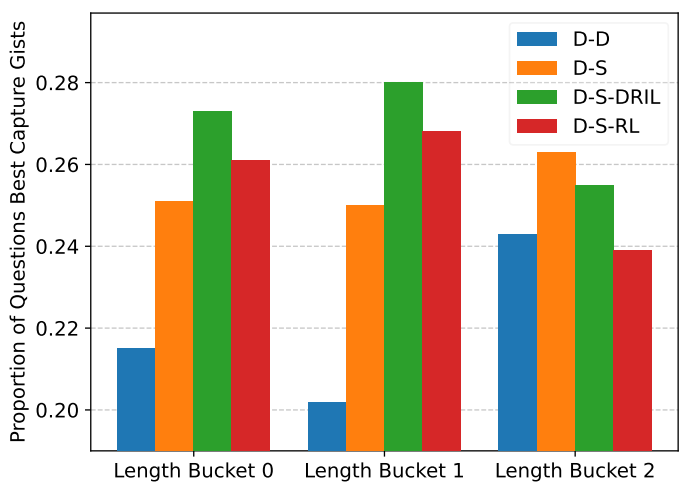

Figure 3: Proportion of most preferred AT-6 questions. (Which question best captures the gist of the article?) According to human evaluation, questions generated by D-S-DRIL best captures the gist of the article in answer length bucket 0 and 1 .

shown in Figure 4, leading to the following observation.

QG models trained on NewsQA and Natural Questions cannot generate self-contained questions that capture gists of articles due to the limitations of the datasets, while QG models trained on $(\mathcal{S C})^{2} \mathcal{Q A}$ can. We can see that the QG model trained on NewsQA achieves about $50 \%$ lower AT-1 accuracy than the other two models, indicating that it cannot generate self-contained questions. Moreover, QG models trained on NewsQA and Natural Questions achieve $73.55 \%$ and $60.03 \%$ lower accuracy on AT-5 (averaged over 3 length buckets) compared with the QG model trained on $(\mathcal{S C})^{2} \mathcal{Q A}$, even though all models generate questions from summaries. We observe that $\mathrm{D}$ S-NewsQA tends to ask trivial questions such as the name of a person. D-S-NQ also fails to identify the focus of a summary. For example, in the summary "Michael Jordan has two brothers and 


\begin{tabular}{|l|c|c|c|c|c|c|}
\hline \multirow{2}{*}{} & \multicolumn{2}{|c|}{ length bucket 0 } & \multicolumn{2}{c|}{ length bucket 1 } & \multicolumn{2}{c|}{ length bucket 2 } \\
\cline { 2 - 7 } & R-L/BLEU & AT-5 Accuracy & R-L/BLEU & AT-5 Accuracy & R-L/BLEU & AT-5 Accuracy \\
\hline D-D & $37.274 / 9.666$ & $0.697 \pm 0.047$ & $37.605 / 10.357$ & $0.736 \pm 0.045$ & $37.643 / 10.688$ & $0.782 \pm 0.042$ \\
\hline D-S & $41.710 / 14.499$ & $0.768 \pm 0.043$ & $41.156 / 13.423$ & $0.782 \pm 0.042$ & $40.489 / 13.174$ & $\mathbf{0 . 8 1 7} \pm \mathbf{0 . 0 4 0}$ \\
\hline D-S-DRIL & $\mathbf{4 2 . 7 6 4 / 1 4 . 8 6 7}$ & $\mathbf{0 . 8 1 4} \pm \mathbf{0 . 0 4 0}$ & $\mathbf{4 1 . 4 4 5 / 1 3 . 6 6 8}$ & $\mathbf{0 . 8 0 6} \pm \mathbf{0 . 0 4 0}$ & $\mathbf{4 0 . 6 7 8 / 1 3 . 7 2 2}$ & $0.809 \pm 0.040$ \\
\hline D-S-RL & $42.596 / 14.756$ & $0.787 \pm 0.042$ & $40.335 / 13.100$ & $0.779 \pm 0.042$ & $40.152 / 12.906$ & $0.815 \pm 0.040$ \\
\hline
\end{tabular}

Table 4: Evaluation of Question Quality. Bold, and bold represents the best results on ROUGE-L(R-L)/BLEU and AT-5 accuracy, respectively. We report a 95\% binomial proportion confidence interval on human evaluation. D-S-DRIL generates significantly better questions in answer length bucket 0 and 1.

\begin{tabular}{|l|c|c|c|}
\hline & length bucket 0 & length bucket 1 & length bucket 2 \\
\hline D-S & $0.566 \pm 0.036$ & $0.670 \pm 0.034$ & $0.693 \pm 0.034$ \\
\hline D-D & $0.521 \pm 0.037$ & $0.614 \pm 0.035$ & $0.681 \pm 0.035$ \\
\hline D-SD & $0.398 \pm 0.072$ & $0.529 \pm 0.075$ & $0.607 \pm 0.073$ \\
\hline QD-D & $0.401 \pm 0.071$ & $0.482 \pm 0.071$ & $0.563 \pm 0.072$ \\
\hline D-S-DRIL & $\mathbf{0 . 5 7 6} \pm \mathbf{0 . 0 3 6}$ & $\mathbf{0 . 6 9 3} \pm \mathbf{0 . 0 3 3}$ & $\mathbf{0 . 7 2 4} \pm \mathbf{0 . 0 3 2}$ \\
\hline D-S-RL & $0.566 \pm 0.040$ & $0.663 \pm 0.038$ & $0.709 \pm 0.037$ \\
\hline \hline CTRLSum & $0.112 \pm 0.046$ & $0.432 \pm 0.073$ & $0.512 \pm 0.076$ \\
\hline QAGen 2S & $0.379 \pm 0.071$ & $\underline{0.514 \pm 0.072}$ & $\underline{0.589 \pm 0.072}$ \\
\hline QA Transfer & $\underline{0.438 \pm 0.140}$ & $0.447 \pm 0.142$ & $0.468 \pm 0.143$ \\
\hline D-S-NewsQA & $0.184 \pm 0.108$ & $0.190 \pm 0.119$ & $0.130 \pm 0.097$ \\
\hline D-S-NQ & $0.118 \pm 0.108$ & $0.171 \pm 0.125$ & $0.226 \pm 0.147$ \\
\hline
\end{tabular}

Table 5: Joint accuracy on AT-1, 2 \& 5. Bold represents our best model and underline represents best baseline. D-S-DRIL generates significantly better QA pairs than the best performing baseline in all three answer length buckets according to the joint AT-1, 2 \& 5 accuracy.

two sisters. He grew up playing basketball and baseball against his older brother.", D-S-NQ generates "Who is Michael Jordan's brother playing against?". However, the summary focus is Michael Jordan rather than his brother. We discuss such cases further in the qualitative analysis section.

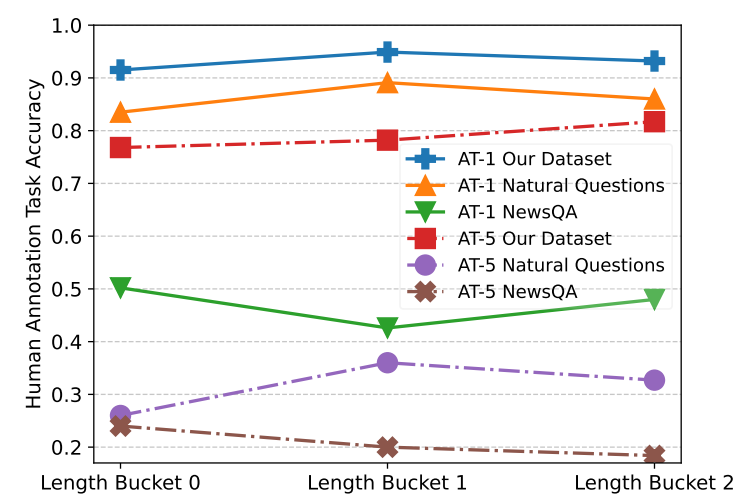

Figure 4: QG Human evaluation on different datasets. Our $(\mathcal{S C})^{2} \mathcal{Q} \mathcal{A}$ dataset performs better than NewsQA and Natural Questions on both AT-1 and AT-5 human evaluation in all three answer length buckets.

\subsection{Overall QA Pair Quality}

We report the joint accuracy of $\{$ AT-1, AT-2, AT$5\}$, defined by the proportion of QA pairs that are

answered TRUE for all three ATs and treat it as a metric for the overall QA pair quality, reporting results in Table 5 with the following observations. D-S-DRIL performs significantly better than the best performing baselines. The best performing baselines are QA Transfer in length bucket 0 and QAGen $2 \mathrm{~S}$ in length bucket 1 and 2. We observe that D-D, D-S, D-S-DRIL and D-S-RL all surpass them by a large margin. Particularly, D-SDRIL outperforms them by $31.51 \%, 34.82 \%$ and $22.92 \%$ in length bucket 0,1 and 2 , respectively.

DRIL consistently outperforms RL and MLE. We can see from Table 5 that D-S-DRIL outperforms D-S and D-S-RL by $3.22 \%$ and $2.80 \%$, respectively (averaged over 3 length buckets). The results are consistent on human annotations (AT-2 in Table 3, AT-5 in Table 4, AT-6 in Figure 3, and joint accuracy in Table 5), and automated metrics (QACS in Table 3 and ROUGE-L/BLEU scores in Table 4). This further shows the advantage of DRIL over MLE and RL, indicating that DRIL can efficiently reinforce AG to generate better QA pairs.

\subsection{Qualitative Analysis}

We also conduct qualitative analyses on generated QA pairs. Please refer to Appendix D for details.

\section{Conclusion}

This paper proposes a model for generating QA pairs with self-contained and summarycentric questions and length-constrained articlesummarizing answers. The target application is suggested questions for conversational news recommendation system. We collect a new dataset, $(\mathcal{S C})^{2} \mathcal{Q A}$, which contains news articles with questions as titles paired with summaries of varying length. We further propose differential reward imitation learning (DRIL) to efficiently mitigate exposure bias encountered with MLE. Empirically, it is shown that DRIL outperforms multiple alternative baseline neural architectures on automated and human evaluations. 


\section{Broader Impact}

Regarding societal considerations, we consider three aspects. (1) Generating QA pairs that correspond to headlines and article summaries to power a news chatbot can provide users with a rapid glance of recent events. However, exposing users exclusively to article summaries may results in less informed users. Naturally, this can be mitigated by also developing experiences that lead to more in-depth examination of articles, but should be carefully considered. (2) Our $(\mathcal{S C})^{2} \mathcal{Q A}$ dataset collection begins with articles (and potentially news providers) that use questions as article titles. Such articles may have stylistic elements that align with certain forms of journalism (e.g., tabloids) or audience manipulation (e.g., alarmism). Accordingly, the corresponding models may learn to generate similarly biased QA pairs which is certainly undesirable. Future work in this direction may include data cleaning to remove biased QA pairs and/or design de-biased models. (3) Factuality is also a potential issue. A news article itself may be fake news. Meanwhile, the AG model may generate a summary that is factually inconsistent with the corresponding news article. Future work may incorporate recent work in optimizing the factual correctness and considering multiple perspectives of the QA pairs.

\section{References}

Chris Alberti, Daniel Andor, Emily Pitler, Jacob Devlin, and Michael Collins. 2019. Synthetic QA corpora generation with roundtrip consistency. In Proceedings of the 57th Annual Meeting of the Association for Computational Linguistics, pages 61686173, Florence, Italy. Association for Computational Linguistics.

Payal Bajaj, Daniel Campos, Nick Craswell, Li Deng, Jianfeng Gao, Xiaodong Liu, Rangan Majumder, Andrew McNamara, Bhaskar Mitra, Tri Nguyen, et al. 2016. Ms marco: A human generated machine reading comprehension dataset. arXiv preprint arXiv:1611.09268.

Massimo Caccia, Lucas Caccia, William Fedus, Hugo Larochelle, Joelle Pineau, and Laurent Charlin. 2019. Language gans falling short. In International Conference on Learning Representations.

Xinya Du and Claire Cardie. 2017. Identifying where to focus in reading comprehension for neural question generation. In Proceedings of the 2017 Conference on Empirical Methods in Natural Language Processing, pages 2067-2073, Copenhagen, Denmark. Association for Computational Linguistics.
Xinya Du and Claire Cardie. 2018. Harvesting paragraph-level question-answer pairs from Wikipedia. In Proceedings of the 56th Annual Meeting of the Association for Computational Linguistics (Volume 1: Long Papers), pages 1907-1917, Melbourne, Australia. Association for Computational Linguistics.

Xinya Du, Junru Shao, and Claire Cardie. 2017. Learning to ask: Neural question generation for reading comprehension. In Proceedings of the 55th Annual Meeting of the Association for Computational Linguistics (Volume 1: Long Papers), pages 1342-1352, Vancouver, Canada. Association for Computational Linguistics.

Junxian He, Wojciech Kryściński, Bryan McCann, Nazneen Rajani, and Caiming Xiong. 2020. Ctrlsum: Towards generic controllable text summarization. arXiv preprint arXiv:2012.04281.

Michael Heilman and Noah A. Smith. 2010. Good question! statistical ranking for question generation. In Human Language Technologies: The 2010 Annual Conference of the North American Chapter of the Association for Computational Linguistics, pages 609-617, Los Angeles, California. Association for Computational Linguistics.

Karl Moritz Hermann, Tomas Kocisky, Edward Grefenstette, Lasse Espeholt, Will Kay, Mustafa Suleyman, and Phil Blunsom. 2015. Teaching machines to read and comprehend. In Advances in Neural Information Processing Systems, volume 28. Curran Associates, Inc.

Ari Holtzman, Jan Buys, Li Du, Maxwell Forbes, and Yejin Choi. 2019. The curious case of neural text degeneration. In International Conference on Learning Representations.

Tom Hosking and Sebastian Riedel. 2019. Evaluating rewards for question generation models. In Proceedings of the 2019 Conference of the North American Chapter of the Association for Computational Linguistics: Human Language Technologies, Volume 1 (Long and Short Papers), pages 2278-2283, Minneapolis, Minnesota. Association for Computational Linguistics.

Ehsan Hosseini-Asl, Bryan McCann, Chien-Sheng Wu, Semih Yavuz, and Richard Socher. 2020. A simple language model for task-oriented dialogue. In $A d$ vances in Neural Information Processing Systems, volume 33, pages 20179-20191. Curran Associates, Inc.

Kalpesh Krishna and Mohit Iyyer. 2019. Generating question-answer hierarchies. In Proceedings of the 57th Annual Meeting of the Association for Computational Linguistics, pages 2321-2334, Florence, Italy. Association for Computational Linguistics.

Tom Kwiatkowski, Jennimaria Palomaki, Olivia Redfield, Michael Collins, Ankur Parikh, Chris Alberti, Danielle Epstein, Illia Polosukhin, Jacob Devlin, Kenton Lee, Kristina Toutanova, Llion Jones, 
Matthew Kelcey, Ming-Wei Chang, Andrew M. Dai, Jakob Uszkoreit, Quoc Le, and Slav Petrov. 2019. Natural questions: A benchmark for question answering research. Transactions of the Association for Computational Linguistics, 7:452-466.

Philippe Laban, John Canny, and Marti A. Hearst. 2020. What's the latest? a question-driven news chatbot. In Proceedings of the 58th Annual Meeting of the Association for Computational Linguistics: System Demonstrations, pages 380-387, Online. Association for Computational Linguistics.

Alex M Lamb, Anirudh Goyal ALIAS PARTH GOYAL, Ying Zhang, Saizheng Zhang, Aaron C Courville, and Yoshua Bengio. 2016. Professor forcing: A new algorithm for training recurrent networks. In Advances in Neural Information Processing Systems, volume 29. Curran Associates, Inc.

Nguyen-Thinh Le, Tomoko Kojiri, and Niels Pinkwart. 2014. Automatic question generation for educational applications-the state of art. In Advanced computational methods for knowledge engineering, pages 325-338. Springer.

Dong Bok Lee, Seanie Lee, Woo Tae Jeong, Donghwan Kim, and Sung Ju Hwang. 2020. Generating diverse and consistent QA pairs from contexts with information-maximizing hierarchical conditional VAEs. In Proceedings of the 58th Annual Meeting of the Association for Computational Linguistics, pages 208-224, Online. Association for Computational Linguistics.

Mike Lewis, Yinhan Liu, Naman Goyal, Marjan Ghazvininejad, Abdelrahman Mohamed, Omer Levy, Veselin Stoyanov, and Luke Zettlemoyer. 2020. BART: Denoising sequence-to-sequence pretraining for natural language generation, translation, and comprehension. In Proceedings of the 58th Annual Meeting of the Association for Computational Linguistics, pages 7871-7880, Online. Association for Computational Linguistics.

Bang Liu, Haojie Wei, Di Niu, Haolan Chen, and Yancheng He. 2020. Asking questions the human way: Scalable question-answer generation from text corpus. In Proceedings of The Web Conference 2020, pages 2032-2043.

Yinhan Liu, Myle Ott, Naman Goyal, Jingfei Du, Mandar Joshi, Danqi Chen, Omer Levy, Mike Lewis, Luke Zettlemoyer, and Veselin Stoyanov. 2019. Roberta: A robustly optimized bert pretraining approach. arXiv preprint arXiv:1907.11692.

Elnaz Nouri, Robert Sim, Adam Fourney, and Ryen W. White. 2020. Proactive suggestion generation: Data and methods for stepwise task assistance. page 1585-1588.

Peng Qi, Yuhao Zhang, and Christopher D. Manning. 2020a. Stay hungry, stay focused: Generating informative and specific questions in information-seeking conversations. In Findings of the Association for Computational Linguistics: EMNLP 2020, pages 25-40, Online. Association for Computational Linguistics.

Weizhen Qi, Yu Yan, Yeyun Gong, Dayiheng Liu, Nan Duan, Jiusheng Chen, Ruofei Zhang, and Ming Zhou. 2020b. ProphetNet: Predicting future n-gram for sequence-to-SequencePre-training. In Findings of the Association for Computational Linguistics: EMNLP 2020, pages 2401-2410, Online. Association for Computational Linguistics.

Pranav Rajpurkar, Robin Jia, and Percy Liang. 2018. Know what you don't know: Unanswerable questions for SQuAD. In Proceedings of the 56th Annual Meeting of the Association for Computational Linguistics (Volume 2: Short Papers), pages 784789, Melbourne, Australia. Association for Computational Linguistics.

Pranav Rajpurkar, Jian Zhang, Konstantin Lopyrev, and Percy Liang. 2016. SQuAD: 100,000+ questions for machine comprehension of text. In Proceedings of the 2016 Conference on Empirical Methods in Natural Language Processing, pages 2383-2392, Austin, Texas. Association for Computational Linguistics.

Marc'Aurelio Ranzato, Sumit Chopra, Michael Auli, and Wojciech Zaremba. 2016. Sequence level training with recurrent neural networks. International Conference on Learning Representations.

Siddharth Reddy, Anca D Dragan, and Sergey Levine. 2020. Sqil: Imitation learning via reinforcement learning with sparse rewards. In 8th International Conference on Learning Representations.

Steven J Rennie, Etienne Marcheret, Youssef Mroueh, Jerret Ross, and Vaibhava Goel. 2017. Self-critical sequence training for image captioning. In Proceedings of the IEEE Conference on Computer Vision and Pattern Recognition, pages 7008-7024.

Siamak Shakeri, Cicero Nogueira dos Santos, Henghui Zhu, Patrick Ng, Feng Nan, Zhiguo Wang, Ramesh Nallapati, and Bing Xiang. 2020. End-to-end synthetic data generation for domain adaptation of question answering systems. In Proceedings of the 2020 Conference on Empirical Methods in Natural Language Processing (EMNLP), pages 5445-5460, Online. Association for Computational Linguistics.

Mohit Shridhar, Jesse Thomason, Daniel Gordon, Yonatan Bisk, Winson Han, Roozbeh Mottaghi, Luke Zettlemoyer, and Dieter Fox. 2020. Alfred: A benchmark for interpreting grounded instructions for everyday tasks. In Proceedings of the IEEE/CVF conference on computer vision and pattern recognition, pages 10740-10749.

Linfeng Song, Zhiguo Wang, Wael Hamza, Yue Zhang, and Daniel Gildea. 2018. Leveraging context information for natural question generation. In Proceedings of the 2018 Conference of the North American Chapter of the Association for Computational 
Linguistics: Human Language Technologies, Volume 2 (Short Papers), pages 569-574, New Orleans, Louisiana. Association for Computational Linguistics.

Yuxuan Song, Ning Miao, Hao Zhou, Lantao Yu, Mingxuan Wang, and Lei Li. 2020. Improving maximum likelihood training for text generation with density ratio estimation. In International Conference on Artificial Intelligence and Statistics, pages 122-132. PMLR.

Nisan Stiennon, Long Ouyang, Jeff Wu, Daniel M Ziegler, Ryan Lowe, Chelsea Voss, Alec Radford, Dario Amodei, and Paul Christiano. 2020. Learning to summarize from human feedback. arXiv preprint arXiv:2009.01325.

Sandeep Subramanian, Tong Wang, Xingdi Yuan, Saizheng Zhang, Adam Trischler, and Yoshua Bengio. 2018. Neural models for key phrase extraction and question generation. In Proceedings of the Workshop on Machine Reading for Question Answering, pages 78-88, Melbourne, Australia. Association for Computational Linguistics.

Xingwu Sun, Jing Liu, Yajuan Lyu, Wei He, Yanjun Ma, and Shi Wang. 2018. Answer-focused and position-aware neural question generation. In Proceedings of the 2018 Conference on Empirical Methods in Natural Language Processing, pages 3930 3939, Brussels, Belgium. Association for Computational Linguistics.

Adam Trischler, Tong Wang, Xingdi Yuan, Justin Harris, Alessandro Sordoni, Philip Bachman, and Kaheer Suleman. 2017. NewsQA: A machine comprehension dataset. In Proceedings of the 2nd Workshop on Representation Learning for NLP, pages 191-200, Vancouver, Canada. Association for Computational Linguistics.

Luu Anh Tuan, Darsh Shah, and Regina Barzilay. 2020 Capturing greater context for question generation. In Proceedings of the AAAI Conference on Artificial Intelligence, volume 34, pages 9065-9072.

Ashish Vaswani, Noam Shazeer, Niki Parmar, Jakob Uszkoreit, Llion Jones, Aidan N Gomez, Ł ukasz Kaiser, and Illia Polosukhin. 2017. Attention is all you need. In Advances in Neural Information Processing Systems, volume 30. Curran Associates, Inc.

Siyuan Wang, Zhongyu Wei, Zhihao Fan, Yang Liu, and Xuanjing Huang. 2019. A multi-agent communication framework for question-worthy phrase extraction and question generation. In Proceedings of the AAAI Conference on Artificial Intelligence, volume 33, pages 7168-7175.

Sean Welleck, Ilia Kulikov, Stephen Roller, Emily Dinan, Kyunghyun Cho, and Jason Weston. 2019. Neural text generation with unlikelihood training. In International Conference on Learning Representations.
Ronald J Williams and David Zipser. 1989. A learning algorithm for continually running fully recurrent neural networks. Neural Computation, 1(2):270 280 .

Xusen Yin, Jonathan May, Li Zhou, and Kevin Small. 2020. Question generation for supporting informational query intents. arXiv preprint arXiv:2010.09692.

Lantao Yu, Weinan Zhang, Jun Wang, and Yong Yu. 2017. Seqgan: Sequence generative adversarial nets with policy gradient. In Proceedings of the AAAI conference on artificial intelligence, volume 31.

Qian Yu, Lidong Bing, Qiong Zhang, Wai Lam, and Luo Si. 2020. Review-based question generation with adaptive instance transfer and augmentation. In Proceedings of the 58th Annual Meeting of the Association for Computational Linguistics, pages 280 290, Online. Association for Computational Linguistics.

Jingqing Zhang, Yao Zhao, Mohammad Saleh, and Peter Liu. 2020. Pegasus: Pre-training with extracted gap-sentences for abstractive summarization. In International Conference on Machine Learning, pages 11328-11339. PMLR.

Yao Zhao, Xiaochuan Ni, Yuanyuan Ding, and Qifa Ke. 2018. Paragraph-level neural question generation with maxout pointer and gated self-attention networks. In Proceedings of the 2018 Conference on Empirical Methods in Natural Language Processing, pages 3901-3910, Brussels, Belgium. Association for Computational Linguistics.

Qingyu Zhou, Nan Yang, Furu Wei, Chuanqi Tan, Hangbo Bao, and Ming Zhou. 2017. Neural question generation from text: A preliminary study. In National CCF Conference on Natural Language Processing and Chinese Computing, pages 662-671. Springer.

Daniel M Ziegler, Nisan Stiennon, Jeffrey Wu, Tom B Brown, Alec Radford, Dario Amodei, Paul Christiano, and Geoffrey Irving. 2019. Fine-tuning language models from human preferences. arXiv preprint arXiv:1909.08593. 


\section{Appendix}

In Appendix A, we describe our data collection procedures. In Appendix B, we describe the training details of each algorithm. In Appendix C, we describe the human evaluation setup on Amazon Mechanical Turk. In Appendix D, we provide qualitative analysis of the generated QA pairs of each model.

\section{A $\quad(\mathcal{S C})^{2} \mathcal{Q} \mathcal{A}$ : A Self-Contained and Summary-Centric QA Dataset}

In this paper, we propose $(\mathcal{S C})^{2} \mathcal{Q A}$, a selfcontained and summary-centric QA dataset. The data construction consists of two steps. First, we collect news articles for which their titles are questions, resulting a set of question-article pairs. Second, for each question in the set, we generate 3 answers that fall into 3 different length buckets. Details are as follows.

\section{A.1 Question-Article Collection}

Starting with a curated URL list of news websites, we mined all articles between September 2020 to March 2021 with the following procedure:

1. For each news article, we check if the title starts with the following words: 'Where', 'What', 'Did', 'Which', 'When', 'How', 'Are', 'Is', 'Can', 'Should', 'Who', 'Will', 'Why', 'Whose', 'Does', 'Do', 'Would', 'Could', 'Shall', 'Was', 'Were', 'Has', 'Have', 'Had'. If not, filter out that article.

2. Then we check if the title ends with '?' and not '??'. If not, filter out that article.

3. If the title matches the following rules, filter out that article: (a) the title includes the word 'you', 'Stock', etc. from an blocklist; (b) the title contain the word 'this' which is not followed by a word in a pre-defined allowlist; (c) the title contains stock symbols. We filter out these titles because these are likely clickbait titles. We also filter out titles that contain punctuation marks beside the question mark at the end, as we want the ground-truth questions to be non-complex sentences.

4. Remove all questions in the articles, as we don't want the model to learn to copy questions from articles.
5. If the number of tokens in an article is less than 100 , or the number of tokens in the title is less than 3 , filter out that article.

In total, we collected 39,461 question-article pairs.

\section{A.2 \{Question, Article, Summary, Length Constraint $\}$ 4-Tuples Collection}

Given the collected question-article pairs, we want to augment them with answers of the questions. We observe that, since the questions are titles of articles, the answers are likely the summaries of articles. From our preliminary study, about $70 \%$ of the questions can be answered by the summaries of the corresponding articles. As a result, we propose to augment the question-article pairs with summaries as pseudo ground truth answers. Unfortunately, not all questions can be answered by the generated summaries, this is because (1) even the ground truth summary may not be the correct answer to the question, (2) summaries predicted by the SoTA models are not necessarily good. Therefore, we need a way to identify if a give summary can answer the corresponding question. This is achieved by training a question-answer classifier.

\section{A.2.1 Question Answer Classifier}

The MS MARCO (Bajaj et al., 2016) dataset contains 4,082,910 labeled question-snippet pairs. A label is either 1 which means that the snippet contains the answer to the question, or 0 which means the snippet does not contain the answer. We finetune a classifier based on RoBERTa-large (Liu et al., 2019) on the MS MARCO dataset. To evaluate how good the trained QA classifier is, we generated around 5000 question-summary pairs, and asked MTurk workers to label whether the summaries answers the corresponding questions. Then we use the trained QA classifier to predict the label.

\begin{tabular}{|l|l|}
\hline AUC & 0.919 \\
\hline Best F1 & 0.960 (P: 0.934, R: 0.989$)$ \\
\hline F1 at Precision=0.98 & 0.903 (P: 0.980, R: 0.837) \\
\hline F1 at Precision=0.97 & 0.937 (P: 0.970, R: 0.906) \\
\hline
\end{tabular}

Table 6: Performance of our QA pair classifier on 5,000 human annotated QA pairs

The performance of our QA pair classifier is shown in Table 6. We can see that the F1 score of the model is 0.96 and when the precision is 0.98 , the recall is 0.903 . This shows that the classifier 
performs sufficiently well for our purposes. Later, we will use this classifier to filter out bad QA pairs. We pick the threshold at which the precision is 0.98 .

\section{A.2.2 The Length of Answers}

For each question, we want to generate three answers, each contain 1, 2 and 3 sentences. Answers with varying length can accommodate different situations such as different screen sizes of voice assistants. Table 7 shows the average number of tokens and characters of the first $\mathrm{K}$ sentences in the ground truth summaries of the CNN/DailyMail dataset. In

\begin{tabular}{|c|c|c|c|c|c|c|c|}
\hline First K sentence & 1 & 2 & 3 & 4 & 5 & 6 & 7 \\
\hline Average \#BPE tokens & 28 & 50 & 72 & 94 & 120 & 140 & 159 \\
\hline Average \#chars & 128 & 232 & 336 & 437 & 558 & 655 & 738 \\
\hline
\end{tabular}

Table 7: Average number of BPE tokens and characters in the first $\mathrm{K}$ sentences of ground truth summaries of the CNN/DailyMail dataset.

our work, we define 3 length buckets for answers with different ranges of number of BPE tokens:

- Length bucket $0:(0,30]$

- Length bucket 1: $(30,50]$

- Length bucket 2: $(50,72]$

Our goal is to be able to specify the length bucket when generate QA pairs, so that we can control the level of brevity for different circumstances (e.g., different screen size of a voice assistant device).

\section{A.2.3 Summary (Answer) Generation}

The high-level idea is to generate summaries using state-of-the-art summarization models under different length constraints and then use the QA pair classifier to filter out unmatched question-summary pairs. The summary generation procedure is shown in Figure 5 and Figure 6. In total, we used four summarization models: PEGASUS (Zhang et al., 2020), BART (Lewis et al., 2020), CTRLSum (He et al., 2020), and ProphetNet (Qi et al., 2020b). All models are fine-tuned on CNNDailyMail dataset. However, we found out ProphetNet Model finetuned on CNN/DailyMail is uncased ${ }^{2}$ so later we removed this model.

For each article, and for each length bucket, we ask each model to generate one summary and we score each question-summary pairs with our QA

\footnotetext{
${ }^{2}$ https://huggingface.co/microsoft/prophetnet-largeuncased-cnndm
}

pair classifier (Note that when generating summaries using CTRLSum, we actually use questions as prompts so that CTRLSum can generate question-conditioned summaries). To ensure that the generated summaries are in the specified length bucket, we enforce summary length via control of the end-of-the-sentence (EOS) token generation. We remove any unfinished sentences at the end, and then reassign a length bucket.

Finally, for each article and each length bucket, we only keep one summary which has the highest score. We also filter out question-summary pairs which have scores below a threshold (which was chosen so that the QA classifier achieves a precision of 0.98 as mentioned earlier in this section). In Table 8, we show the number of summaries generated by each model and accepted by our selection strategy. In the future, one could easily introduce more SoTA summarization models in the dataset generation process. Finally, we generate a dataset containing 53,746 entries. Each entry contains the following components: question, article, summary, length bucket, QA pair classifier score, model source. Length bucket is an enumerated type consisting of 'LB0', 'LB1' and 'LB2'. Model source is also an enumerated type consisting of 'PEGASUS', 'BART' and 'CTRLSum'. Table 9 shows the number of BPE tokens and the number of characters of the summaries in each length bucket. Each cell's format is \#BPE/\#char.

Figure 7 compares the distributions of the first word of a question in $(\mathcal{S C})^{2} \mathcal{Q A}$, NewsQA, Natural Questions, and SQuAD (Rajpurkar et al., 2018) dataset. As we can see, $(\mathcal{S C})^{2} \mathcal{Q A}$ is more diverse in terms of the first words in questions.

\section{A.3 Examples of the Data}

Tables 10 - 13 show 4 examples in our dataset.

\section{B Training Details}

We use Pytorch and the Transformers package ${ }^{3}$ to implement our algorithms and baselines. The AG models of all the algorithms are initialized by a pre-trained DistilBART model that is fine-tuned on the CNN/DailyMail dataset, ${ }^{4}$ and the QG models of all the algorithms are intialized by a pre-trained DistilBART model that is fine-tuned on the XSum dataset. ${ }^{5}$ For these two pre-trained models, the

\footnotetext{
${ }^{3}$ https://huggingface.co/transformers/

${ }^{4}$ https://huggingface.co/sshleifer/distilbart-cnn-12-6

${ }^{5}$ https://huggingface.co/sshleifer/distilbart-xsum-12-6
} 


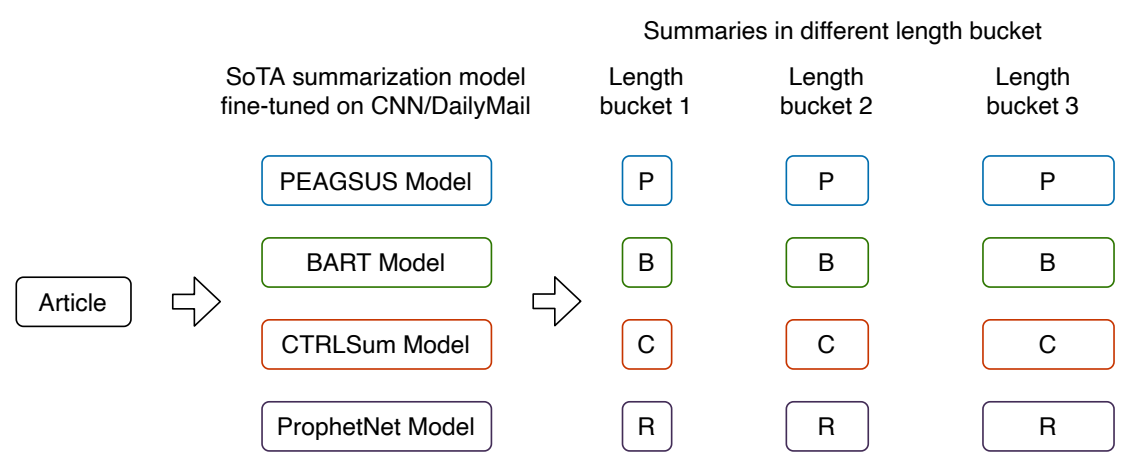

Figure 5: SoTA summarization models are used to generate summaries under each length bucket constraints. P, B, $\mathrm{C}$, and R represent the summaries generated by PEAGSUS, BART, CTRLSum and ProphetNet model, respectively.

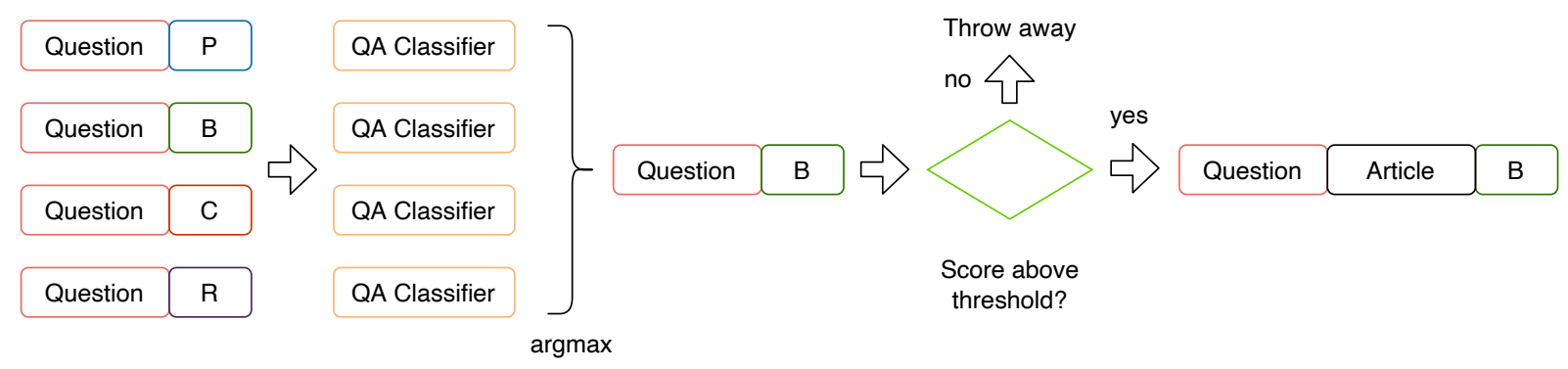

Figure 6: Summaries are then scored by the QA pair classifier. The one with the highest score that is also higher than the threshold is kept.

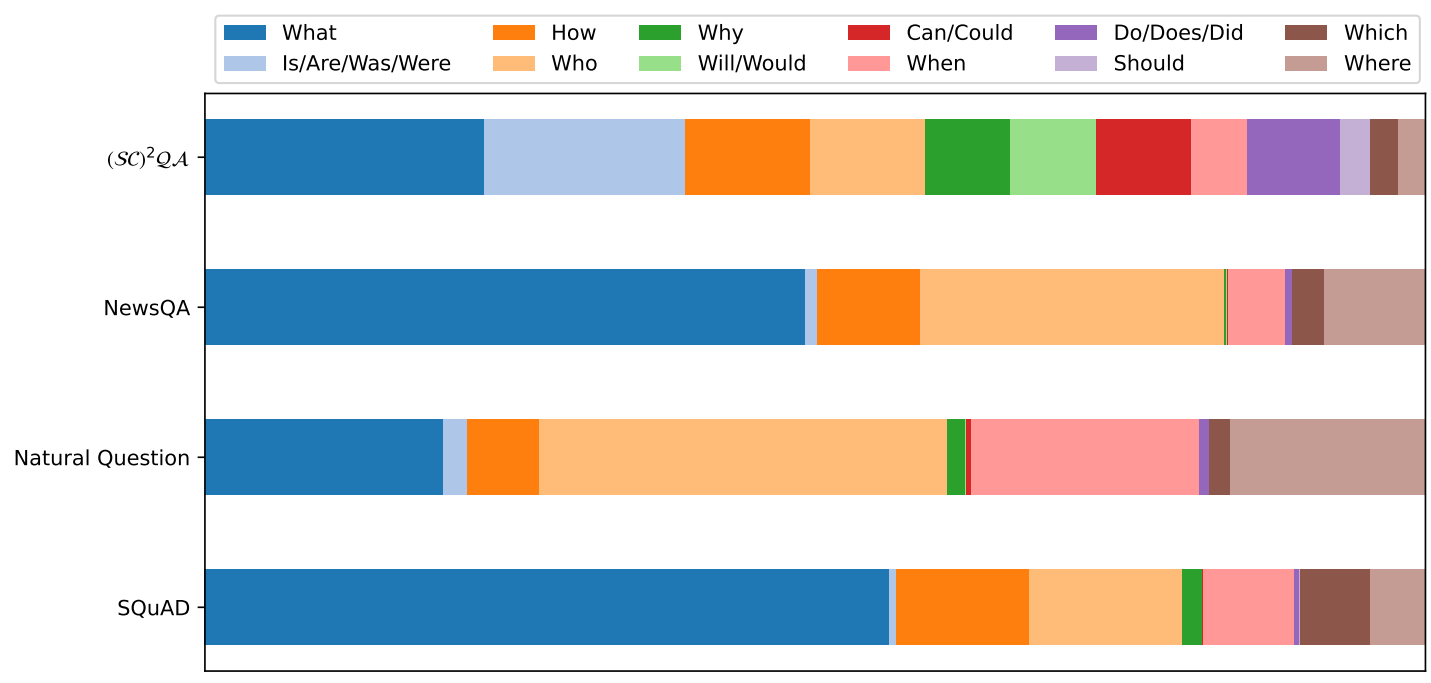

Figure 7: Each horizontal bar represents a distribution of the first word of a question in a dataset. Each color represents the proportion of the corresponding word as the first word in a question. The 12 words shown here are the most frequent first words in all the dataset. This figure shows that $(\mathcal{S C})^{2} \mathcal{Q} \mathcal{A}$ has more diverse initial words of questions.

\begin{tabular}{|l|c|c|c|c|c|c|c|c|c|}
\hline & \multicolumn{3}{|c|}{ length bucket 0: \#BPE $(0,30]$} & \multicolumn{3}{c|}{ length bucket 1: \#BPE $(30,50]$} & \multicolumn{3}{c|}{ length bucket 2: \#BPE (50, 72] } \\
\hline & PEGASUS & BART & CTRLSum & PEGASUS & BART & CTRLSum & PEGASUS & BART & CTRLSum \\
\hline \#summaries fall in this bucket & 32618 & 31736 & 35638 & 31513 & 30880 & 35030 & 32700 & 34608 & 35719 \\
\hline \#summaries accepted & 4112 & 3343 & 4243 & 5542 & 5318 & 7665 & 6807 & 7608 & 9107 \\
\hline acceptance rate & 0.126 & 0.105 & 0.119 & 0.176 & 0.172 & 0.219 & 0.208 & 0.220 & 0.255 \\
\hline proportion in the dataset & 0.352 & 0.286 & 0.363 & 0.299 & 0.287 & 0.414 & 0.289 & 0.323 & 0.387 \\
\hline
\end{tabular}

Table 8: Statistics of summaries generated by each SoTA summarization model in different length buckets. 


\begin{tabular}{|l|c|c|c|c|c|c|}
\hline & min length & max length & mean length & median length & $10 \%$ percentile length & $90 \%$ percentile length \\
\hline length bucket 0 & $10 / 32$ & $32 / 217$ & $24.49 / 105.60$ & $25 / 104$ & $18 / 70$ & $31 / 143$ \\
\hline length bucket 1 & $33 / 88$ & $52 / 339$ & $43.56 / 197.29$ & $44 / 197$ & $36 / 153$ & $51 / 242$ \\
\hline length bucket 2 & $53 / 167$ & $74 / 454$ & $63.98 / 295.18$ & $64 / 293$ & $56 / 224$ & $72 / 349$ \\
\hline
\end{tabular}

Table 9: Number of BPE tokens and number of characters of the summaries in each length bucket. Each cell's format is \#BPE/\#char.

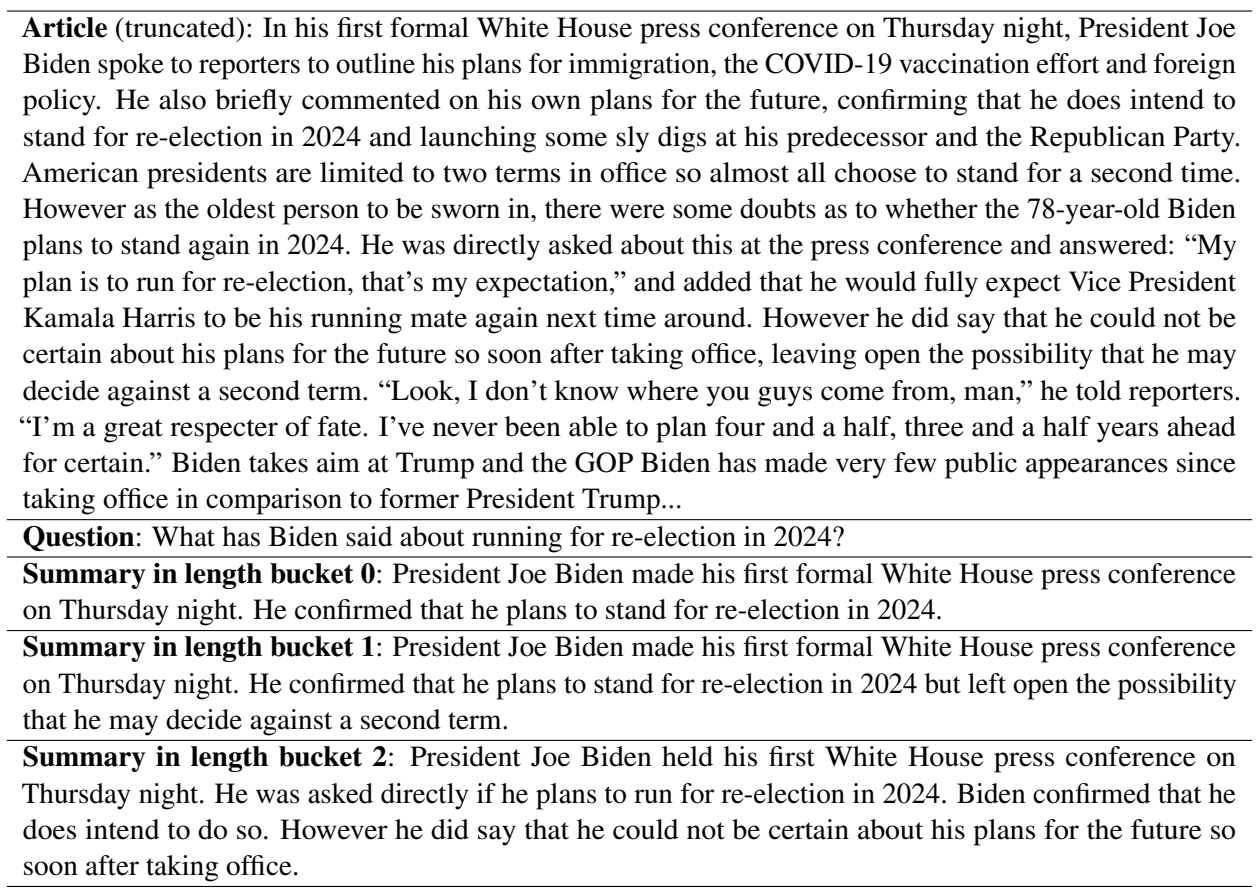

Table 10: Question-Article-Summary-Length Bucket example 1/5.

number of encoder layers is 12 , the number of decoder layers is 6 , the dimension of hidden states is 1,024 , and the number of attention head is 16 .

All the experiments are conducted on AWS EC2 p3dn.24xlarge GPU instances and run with 8 GPUs in parallel. We use the Seq2SeqTrainer from the Transformers package to control the training process. Hyper-parameters are selected based on the ROUGE-L score on validation set described previously (the last 5,000 entries of the data we generated). All the models are optimized with Adam with linear learning rate scheduling, and the number of warm up steps is 500 . All the batch sizes are set to 8 . The number of beams during inference is set to 4 .

D-S. The QG model's learning rate is $2 \times 10^{-5}$ and the number of iterations is 5 . The AG model's learning rate is $2 \times 10^{-5}$ and the number of iterations is 10 .

D-D. The QG model's learning rate is $3 \times 10^{-5}$ and the number of iterations is 10 . The AG model is the same as D-S's AG model.

D-SD. The QG model's learning rate is $3 \times 10^{-5}$ and the number of iterations is 5 . The AG model is the same as D-S's AG model.

QD-D. The QG model's learning rate is $3 \times 10^{-5}$ and the number of iterations is 10 . The AG model's learning rate is $2 \times 10^{-5}$ and the number of iterations is 10 .

D-S-DRIL. The QG model is the same as D-S's QG model. The AG model's learning rate is $3 \times$ $10^{-5}$ and the number of iterations is 10 . Moreover, as we described in the paper, for the AG model we optimize the sum of DRIL loss and cross entropy loss, and we set $\lambda$ (the weight of the DRIL loss) to 0.3 .

D-S-RL. The QG model is the same as D-S's QG model. The reward model for AG is a copy of the QG model and is fixed during training. The reward model calculates the negative log-likelihood of a generated question given a generated summary. We use self-critic (Rennie et al., 2017) to train D-S-RL. The learning rate is $2 \times 10^{-5}$ and the number of iterations is 10. Similar to D-S-DRIL, we optimize the sum of RL loss and cross entropy loss, and $\lambda$ (the weight of the RL loss) is set to 0.1 . 


\begin{abstract}
Article (truncated): Public health officials say it's important to vaccinate as many people as quickly as possible to reduce the risk posed by new coronavirus variants. One strategy to stretch existing supplies albeit with huge logistical challenges would be to give just one dose of the vaccine to people who have recovered from COVID-19. About half a dozen small studies, all consistent with one another but as yet unpublished, suggest this strategy could work. Dr. Mohammad Sajadi, at the University of Maryland medical school's Institute of Human Virology studied health care workers who were just getting their first of two vaccine shots. His research team homed in on those who had previously been diagnosed with COVID-19. "We saw a much faster response and a much higher response," he says, based on the protective antibodies his team measured in the blood. The infection served the same priming role as an initial dose of the Moderna or Pfizer vaccine would have, so the first shot they got was in effect a booster. It amplified and solidified immunity to COVID-19. The study was published Monday in JAMA, the journal of the American Medical Association. The Johnson \& Johnson vaccine authorized Saturday by the Food and Drug Administration only requires a single dose. So, he says while vaccine is scarce, it makes sense to offer just one shot to people who have already had the disease. "You can free up automatically millions of doses," he says, increasing vaccine supply by 4 percent or 5 percent. "We think it makes sense at this time to promote such a policy." Federal health officials are intrigued. Dr. Anthony Fauci, who serves as COVID-19 adviser to the White House, has said it's an idea worth further study. He is dead set against another strategy, which is stretching out the time between first and second doses. But health officials are not ready to say yes...

Question: Could a single-dose of COVID-19 vaccine after illness stretch the supply?

Summary in length bucket 0: One strategy to stretch existing supplies would be to give just one dose of the vaccine to people who have recovered from COVID-19.

Summary in length bucket 1: One strategy to stretch existing supplies would be to give just one dose of the vaccine to people who have recovered from COVID-19. About half a dozen small studies suggest this strategy could work.

Summary in length bucket 2: One strategy to stretch existing supplies would be to give just one dose of the vaccine to people who have recovered from COVID-19. About half a dozen small studies suggest this strategy could work. Federal health officials are intrigued, but are not ready to say yes.
\end{abstract}

Table 11: Question-Article-Summary-Length Bucket example 2/5.

QAGen 2S. The learning rate of both the $\mathrm{QG}$ and AG model is $2 \times 10^{-5}$ and the number of iterations is 10 . See Table 15 for training and inference pipelines.

CTRLSum. The QG model is the same as QDD's QG model. The AG model is the officially pre-trained CTRLSum model. ${ }^{6}$ When generating question-conditioned summaries (answers) using the pre-trained CTRLSum model, we use the questions as prompts. See Table 15 for training and inference pipelines.

QA Transfer. The QG model is the same as QDD's QG model. The AG model is trained on the NewsQA dataset. Since the provided answers in NewsQA dataset are short spans of text, we treat the sentences that contain the answer spans as ground truth answers. The input of the encoder is a concatenation of a question and an article, separated by $</ s\rangle$, and the label of the decoder is the ground truth answer. The learning rate is $2 \times 10^{-5}$ and the number of iterations is 10 . See Table 15 for training and inference pipelines.

D-S-NewsQA. The QG model is trained on the NewsQA dataset. The input of the encoder is an article, and the label of the decoder is a question.

\footnotetext{
${ }^{6}$ https://github.com/salesforce/ctrl-sum
}

The learning rate is $2 \times 10^{-5}$ and the number of iterations is 10. The AG model is the same as D-S's AG model. During inference, questions are generated from summaries. See Table 15 for training and inference pipelines.

D-S-NQ. The QG model is trained on the Natural Questions dataset. The input of the encoder is a long answer, and the label of the decoder is a question. The learning rate is $2 \times 10^{-5}$ and the number of iterations is 10 . The AG model is the same as D-S's AG model. During inference, questions are generated from summaries. See Table 15 for training and inference pipelines.

\section{Human Evaluation Setup}

We used Amazon Mechanical Turk to conduct human evaluations. In total we completed two rounds of annotation. In round 1, we evaluated a QA pair generated by a model. The task layout for round 1 is shown in Figure 8. Each human intelligence task (HIT) has 5 tasks. First, a QA pair is shown. Task 1 (AT-1) asks if the question is self-contained; Task 2 (AT-2) asks if the answer answers the question; Task 3 (AT-3) asks if the answer is both succinct and sufficient; Task 4 (AT-4) asks the annotator to select a span of the answer that is succinct and suf- 


\begin{abstract}
Article (truncated): Find out in which countries and after what cases vaccination is stopped, what scientists and officials say about the relationship between AstraZeneca and thrombosis, and how the pharmaceutical company itself responded. More than a dozen countries, mostly in the European Union, have suspended the use of the AstraZeneca Covid-19 vaccine due to concerns that some patients have developed blood clots. The World Health Organization (WHO) urged countries to continue using the vaccine, but still decided to convene a meeting due to the massive halt in AstraZeneca vaccination. In total, about 17 million people have received AstraZeneca vaccinations (at least one dose) in the European Union and the UK. Among them, 40 people had blood clots after vaccination. Whether the AstraZeneca vaccine is related to thrombosis is not clear, since its use is not long enough. Vaccine advocates argue that the drug can be used, and the proportion of patients with thrombosis is consistent with the usual statistics, and the vaccine has nothing to do with it. At the same time, many governments have decided to suspend (rather than ban entirely) the vaccination of AstraZeneca pending an investigation by the EMA regulator and estimates by WHO experts. Which countries have suspended vaccination Denmark became the first country to stop using the AstraZeneca Covid-19 vaccine for two weeks after reports of blood clots in some people and even one death on 11 March. A 60-year-old woman who was vaccinated with AstraZeneca developed a blood clot and died. She was vaccinated from the same batch used in Austria. During these two weeks of suspension of vaccinations, the EMA is to investigate. Norway, Iceland, Luxembourg, Romania, and Congo followed Denmark's example. Norwegian authorities said Saturday that four people under 50 who received the AstraZeneca vaccine had unusually low platelet counts in their blood, which could lead to severe bleeding. Bulgaria on March 12 suspended the use of the drug after the death of a 57-year-old woman a few hours after vaccination...

Question: Why major European nations suspend use of AstraZeneca vaccine?

Summary in length bucket 0 : -

Summary in length bucket 1: More than a dozen countries, mostly in the European Union, have suspended the use of the AstraZeneca Covid-19 vaccine due to concerns that some patients have developed blood clots.

Summary in length bucket 2: More than a dozen countries, mostly in the European Union, have suspended the use of the AstraZeneca Covid-19 vaccine due to concerns that some patients have developed blood clots. The World Health Organization urged countries to continue using the vaccine, but still decided to convene a meeting due to the massive halt.
\end{abstract}

Table 12: Question-Article-Summary-Length Bucket example 3/5.

ficient (This task enforces the annotator to read the answers carefully). Following Task 4, we show the corresponding article. Then, Task 5 (AT-5) asks if the question captures the gist of the article.

Each HIT has 3 assignments, that is, each HIT will be annotated by 3 different annotators. We used majority vote to aggregate annotations. We designed a qualification task which contains 5 HITs with their annotations determined by the authors of this paper. We qualified annotators who had an accuracy (using annotations from the authors of this paper as ground truth labels) greater than or equal to $80 \%$. We observed that on average it took about 2 minutes to annotate one HIT. We paid $\$ 0.35$ per HIT with a $\$ 0.1$ bonus. We blocked annotators who spent less than 1 minutes on average on a HIT. If an annotator was blocked, then all the annotations from that annotator were thrown away.

The annotation results in length bucket 0,1 , and 2 are shown in Tables $16-18$, respectively. In total, we have 11 algorithms. During round 1, we realized that some algorithms performed significantly worse than the others, so there is no reason to collect the equal amount of HITs for every algorithm. Therefore, the number of completed hits for each algorithm is different, as shown in the "completed HITs' columns of Tables 16 - 18. Meanwhile, since we filtered out annotations from blocked annotators, this also led to different numbers of completed hits between different models. During round 1, we did 7 mini-round annotations in total (each between 50 to 150 HITs), and in the last 3 mini-rounds AT5 was excluded. When AT-5 was excluded, the annotators did not need to read the article, so the annotation process was accelerated and we were able to collect more annotations for AT- 1 to AT-4.

From round 1 we observed that D-S, D-D, D-SDRIL, and D-S-RL perform the best. Therefore, we conducted annotation round 2 , which compared the questions generated by these four models in one HIT. The task layout for round 2 is shown in Figure 9. We first show an article, and then show the questions generated by each model. If two or more questions generated by different models are the same, we then merge these questions into one. Therefore, we show 2 to 4 questions in one HIT. We randomly shuffle the order of the questions in each HIT, so that the question of a model can appear in any position. Task 1 in round 2 (corresponding to AT-5) asks if each of the question captures the 


\begin{abstract}
Article (truncated): Britain's royal family is among the world's most famous organizations - and a costly one as well. These days, the royal family is known for their lavish weddings, expansive tours and notable fashion as much as they are for their contributions to their nation. According to the BBC, the royals amass their fortune, in part, through the taxpayer-funded Sovereign Grant. However, the queen and the other royals get the money in return for surrendering the profits from their slew of properties - called the Crown Estate - to the government, according to Business Insider. Each year, the queen will receive an amount from the grant equivalent to $25 \%$ of the Crown Estate's profits, the outlet reports. The grant will pay for the palace upkeep, the family's travel, royal employee payroll and more, but according to the Telegraph, the Grant doesn't cover costs for security and royal ceremonies, per BI. Money for such assets and events comes from a portfolio of land that the family has owned for generations called the Duchy of Lancaster. The Duchy is made up of residential, commercial, and agricultural properties, Insider reports, and contains $\$ 715$ million worth of net assets. In 2019, the portfolio earned \$27 million, The Wall Street Journal reports. The money is put toward 'expenses incurred by other members of the royal family,' as the royal family's website puts it...

Question: Where does the royal family get their money?

Summary in length bucket 0: Britain's royal family amass their fortune, in part, through the taxpayerfunded Sovereign Grant.

Summary in length bucket 1: Britain's royal family amass their fortune, in part, through the taxpayerfunded Sovereign Grant. The queen and the other royals get the money in return for surrendering the profits from their slew of properties.

Summary in length bucket 2: Britain's royal family amass their fortune, in part, through the taxpayerfunded Sovereign Grant. The queen and other royals get the money in return for surrendering the profits from their slew of properties to the government. Money for such assets and events comes from a portfolio of land that the family has owned for generations called the Duchy of Lancaster.
\end{abstract}

Table 13: Question-Article-Summary-Length Bucket example 4/5.

gist of the article; Task 2 in round 2 (corresponding to AT-6) asks which question best capture the gist of the article; Task 3 in round 2 (corresponding to AT-7) asks which question is preferred if suggested by a voice assistant in a news skill. The annotation results in length bucket 0,1 , and 2 are shown in Table $19-21$. While round 1 and round 2 both have AT-5, we observe that the three algorithm (D-S, DD, D-S-DRIL) have lower AT-5 accuracy in round 2 than in round 1 . We believe that this is because the round 2 task layout better encourages a more careful reading of the articles by the annotators. However, pairwise preference of AT-5 accuracy is consistent between round 1 and round 2 . 
Article (truncated): NASA's Perseverance rover and its sibling, the Ingenuity helicopter, landed on Mars on February 18, bristling with antennas and cameras. Perseverance, the third robotic visitor from Earth to arrive at the red planet, will spend the next Martian year the equivalent of two Earth years collecting rocks, scrutinizing and photographing them. But the \$2.7-billion robotic explorer has one thing in common with something closer home. The rover has the same processor as the original iMac G3 or the 'Bondi Blue' from 1998. The original iMac used a PowerPC G3 or the PowerPC 750 processor which mirrors the one used in Perseverance, said a report in The Verge. The processor, a single-core, $233 \mathrm{MHz}$ processor with just 6 million transistors, was also used in NASA's Curiosity rover, a car-sized rover exploring the red planet which was launched in 2011. The report says that the conditions on Mars could actually be counterproductive for a more advanced processor. Compared to Earth's atmosphere, the atmosphere on the red planet does not offer as much insulation from harmful radiation and charged particles. This could mess up a modern, more complex processor. The Perseverance rover has two computing modules, one being a backup in case of a mishap. Perseverance's processor, a RAD750 chip, is slightly more advanced than the one used in the iMac G3 and is built keeping Mars's radiations in mind. It operates at up to 200 megahertz speed, 10 times the speed in Mars rovers Spirit and Opportunity's computers. Coming to memory power, Perseverance boasts 2 gigabytes of flash memory, 256 megabytes of dynamic random access memory (RAM), and 256 kilobytes of electrically erasable programmable read-only memory. The computer also contains special memory to tolerate the extreme radiation environment that exists in space and on the Martian surface, says NASA...

Question: What do NASA's Mars rover and a 1998 iMac have in common?

Summary in length bucket 0: The rover has the same processor as the original iMac G3 or the 'Bondi Blue' from 1998.

Summary in length bucket 1: Perseverance rover has same processor as the original iMac G3 or the 'Bondi Blue' from 1998. The processor was also used in NASA's Curiosity rover, a car-sized rover, launched in 2011.

Summary in length bucket 2: The rover has the same processor as the original iMac G3 or the 'Bondi Blue' from 1998. NASA's Perseverance rover has two computing modules, one being a backup in case of a mishap. The computer also contains special memory to tolerate the extreme radiation environment that exists in space and on the Martian surface, says NASA.

Table 14: Question-Article-Summary-Length Bucket example 5/5.

\begin{tabular}{|c|c|c|c|c|c|c|c|c|}
\hline & \multicolumn{4}{|c|}{ Training } & \multicolumn{4}{|c|}{ Inference } \\
\hline & \multicolumn{2}{|c|}{ Answer Generation } & \multicolumn{2}{|c|}{ Question Generation } & \multicolumn{2}{|c|}{ Answer Generation } & \multicolumn{2}{|c|}{ Question Generation } \\
\hline & Encoder & Decoder & Encoder & Decoder & Encoder & Decoder & Encoder & Decoder \\
\hline D-S & $L+D$ & $\mathrm{~S}$ & $\mathrm{~S}$ & $\mathrm{Q}$ & $\mathrm{L}+\mathrm{D}$ & S' & S' & Q' \\
\hline D-D & $\mathrm{L}+\mathrm{D}$ & $\mathrm{S}$ & $\mathrm{D}$ & Q & $\mathrm{L}+\mathrm{D}$ & $S^{\prime}$ & $S^{\prime}$ & $Q^{\prime}$ \\
\hline D-SD & $\mathrm{L}+\mathrm{D}$ & $\mathrm{S}$ & $\mathrm{S}+\mathrm{D}$ & Q & $\mathrm{L}+\mathrm{D}$ & $S^{\prime}$ & $S^{\prime}+D$ & $Q^{\prime}$ \\
\hline QD-D & $Q+L+D$ & $\mathrm{~S}$ & $\mathrm{D}$ & $\mathrm{Q}$ & $Q^{\prime}+\mathrm{L}+\mathrm{D}$ & S' & $\mathrm{D}$ & $\mathrm{Q}^{\prime}$ \\
\hline D-S-DRIL & $\mathrm{L}+\mathrm{D}$ & S/S' & $\mathrm{S}$ & $\mathrm{Q}$ & $\mathrm{L}+\mathrm{D}$ & $\mathrm{S}^{\prime}$ & $S^{\prime}$ & $Q^{\prime}$ \\
\hline D-S-RL & $\mathrm{L}+\mathrm{D}$ & S/S' & $\mathrm{S}$ & Q & $\mathrm{L}+\mathrm{D}$ & $S^{\prime}$ & $S^{\prime}$ & $Q^{\prime}$ \\
\hline QAGen 2S & $\overline{\mathrm{D}+\mathrm{Q}}$ & S & $\overline{\mathrm{D}}$ & $\overline{Q Q}$ & $\overline{D+Q^{\prime}}$ & S' & $\overline{\mathrm{D}}$ & $\mathrm{Q}^{\prime}$ \\
\hline CTRLSum & Pretrained CTR & Sum model & $\mathrm{D}$ & Q & $Q^{\prime}+\mathrm{D}$ & $S^{\prime}$ & $\mathrm{D}$ & $Q^{\prime}$ \\
\hline QA Transfer & $\mathrm{Q}+\mathrm{D}$ in NewsQA & A in NewsQA & $\mathrm{D}$ & Q & $Q^{\prime}+D^{\prime}$ & $\mathrm{A}^{\prime}$ & $\mathrm{D}$ & $Q^{\prime}$ \\
\hline D-S-NewsQA & $\mathrm{L}+\mathrm{D}$ & $S$ & D in NewsQA & Q in NewsQA & $\mathrm{L}+\mathrm{D}$ & $S^{\prime}$ & $S^{\prime}$ & $Q^{\prime}$ \\
\hline D-S-NQ & $\mathrm{L}+\mathrm{D}$ & $S$ & LA in Natural Questions & Q in Natural Questions & $\mathrm{L}+\mathrm{D}$ & $S^{\prime}$ & $S^{\prime}$ & $Q^{\prime}$ \\
\hline
\end{tabular}

Table 15: A summary of our models and baselines. Q, S, D, L denote the questions, summaries, documents, and length bucket tags in our dataset, respectively. Q' and S' denote the generated questions and answers, respectively. D in NewsQA, Q in NewsQA, and A in NewsQA denote the documents, questions, and answers in the NewsQA dataset. A' denotes the answers generated by the QA model in QA Transfer. Q + D in NewsQA denotes the concatenation of questions and documents in the NewsQA dataset with $\langle/ s\rangle$ as the separator. LA in Natural Questions and Q in Natural Questions denote the long answers and questions in the Natural Questions dataset, respectively. 


\section{Instruction}

In this assignment, you will measure the quality of a question-answer pair generated for a specific article. First, you are given the question and the answer, and you need to answer four questions regarding whether the question is understandable and whether the answer sufficiently and succinctly answers the question. Then, you are given the article, and you need to identify if the question refers to the gist of the article. (Detailed Instructions are shown below)

Please read the question and the answer below to finish task 1-4 and then read the article to finish task 5.

Question:

Why Did Energy Monster's Shares Jump 18\% on Their Nasdaq Debut?

Answer:

Shares of Alibaba-backed Energy Monster jumped nearly $18 \%$ in their Nasdaq debut on Thursday. The companys initial public offering (IPO) was priced below the indicated range. Energy Monster was embroiled in an ownership dispute just as the U.S. IPO was underway.

Task 1: Is the question self-contained (without referring to the answer or the article, are you able to understand the question)?

Task Instruction:

Some questions such as "Why leave town?", "What country was the foreign agent from?" and "What did scientists say?" are not self-contained and to understand what the questions ask exactly, we have to refer to the articles. Some other questions such as "What are educator's legal rights if they go back to work during the coroanvirus pandemic?" and "How Good Was NCAA Tournament Analyst Wally Szczerbiak as a
Player?" are self-contained questions and can be understood without referring to the article.

O True $O$ False

Task 2: Does the passage in the Answer text box answers the question?

Task Instruction:

Imagine that you ask this question to a voice assistant (e.g., Amazon Alexa, Google Assistant, Apple Siri) and the passage in the Answer text box is returned. Do you think the Answer satisfactorily answers the corresponding Ouestion?

O True $O$ False

\section{Task 3: To confidently trust the answer, select one of the following}

Task Instruction:

Again, imagine that you ask this question to a voice assistant (e.g., Amazon Alexa, Google Assistant, Apple Siri) and the passage in the Answe text box is returned.

(a) I would need to see more of the article to trust the Answer (i.e., it is not sufficient).

(b) There is more content in the passage in the Answer text box than necessary (i.e., it is not succinct).

$O$ (c) The Answer is both succinct and sufficient.

Task 4: If the answer to Task 3 is (b), please copy the substring in the Answer text box that you think is succinct and sufficient.

task4 answer

Please continue by reading the (truncated) article below.

Article:

HONG KONG (Reuters) - Shares of Alibaba-backed Energy Monster, Chinas largest provider of mobile charging devices, jumped nearly $18 \%$ in their Nasdaq debut on Thursday after the companys initial public offering (IPO) was priced below the indicated range.

The firms shares opened at $\$ 10$ per American depositary share (ADS), higher than the IPO price of $\$ 8.50$ per ADS.

The company, which filed its prospectus under the listing vehicles name of Smart Share Global, sold 17.65 million shares at $\$ 8.50$ each to raise about $\$ 150$ million.

Energy Monsters weaker pricing comes just days after Chinese question and answer website Zhihus shares fell as much as $10.5 \%$ in their first session on Friday. The stock is off nearly $15 \%$ since its debut on the New York Stock Exchange.

Y. IPO was underway. Energy Monster had flagged its shares would be sold between $\$ 10.50$ and $\$ 12.50$ each.

vestors would pay for the shares.

There are also ongoing concerns over the future of Chinese companies listed in the United States following the Securities and Exchange Commissions decision to press ahead with laws that would see international companies that do not meet U.S. auditing standards delisted. 政 $\$ 183.75$ million to $\$ 218.7$ million at the indicative range. Two Shanghai-based venture capitalists are pressing a case through both U.S. and Chinese courts against Energy Monster Chief Executive Officer Guangyuan Cai, claiming he reneged on a deal to give them a joint 3\% stake in the business. frivolous, and the CEO is contesting the claims vigorously.

Task 5: Does the question in the Question text box (copied here for convenience) capture the gist of the Article?

Question:

Why Did Energy Monster's Shares Jump 18\% on Their Nasdaq Debut?

Task Instruction

Please only rely on the truncated text of the Article but not other information such as images or audio (if they exist) to finish this task.

$O$ Yes $O$ No

Figure 8: Human annblation UI for round 1. 


\section{Instruction}

In this assignment, you are given an article and 1 4 questions, and you need to identify if each question capture the gist of the article. (Detailed Instructions are shown below)

\section{Please read the (truncated) article below.}

Article:

(Reuters) - Microsoft Corp said on Thursday it was investigating an issue with its Microsoft 365 services and features, including workplace messaging app Teams and Azure, after many users were unable to access them.

Outage tracking website Downdetector showed over 8,000 incidents of people reporting issues with Teams, an app heavily relied on by people for remote work during the COVID-19

pandemic.

"We're investigating an issue in which users may be unable to access Microsoft 365 services and feares. We'll provide additional information as soon as possible" said Microsoft in a tweet

Downdetector only tracks outages by collating status reports from a series of sources, including user-submitted errors on its platform. The outage might be affecting a larger number of users.

Task 1: Does the question in the Question text box capture the gist of the Article?

Task Instruction: Please only rely on the truncated text of the Article but not other information such as images or audio (if they exist) to finish this task.

Question 1: Is Microsoft Teams outage affecting a larger number of users?

$$
\mathrm{O} \text { Yes } \mathrm{O} \text { No }
$$

Question 2: What's going on with Microsoft Teams and Azure?

$$
\mathrm{O} \text { Yes } \mathrm{O} \text { No }
$$

Question 3: Why isn't Teams working?

$$
\mathrm{O} \text { Yes } \mathrm{O} \text { No }
$$

Question 4: What's going on with Microsoft 365?

$$
\text { O Yes O No }
$$

Task 2: Which Question shown above best capture the gist of the Article?

O Question $1 \quad O$ Question $2 \quad O$ Question $3 \quad O$ Question 4

Task 3: If these $\mathbf{4}$ questions are suggested by a news skill of a voice assistant (e.g. Amazon Alexa, Apple Siri, etc.) on the screen, which question would you be most likely to ask?

O Question 1 Q question $2 \quad \mathrm{Q}$ Question $3 \quad \mathrm{O}$ Question 4

Figure 9: Human annotation UI for round 2. Here Task 1 corresponds to AT-5 (same as Task 5 in round 1), Task 2 corresponds to AT-6 and Task 3 corresponds to AT-7. 


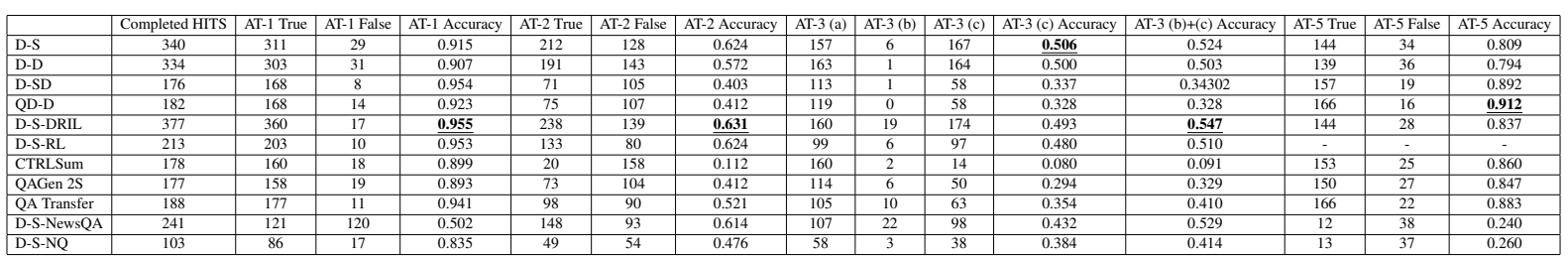

Table 16: Round 1 length bucket 0 human annotation. AT-5 annotation for D-S-RL will be in Round 2.

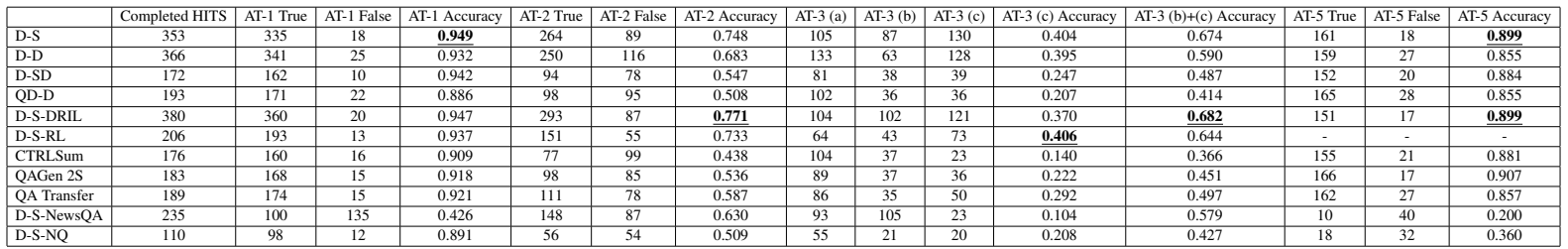

Table 17: Round 1 length bucket 1 human annotation. AT-5 annotation for D-S-RL will be in Round 2.

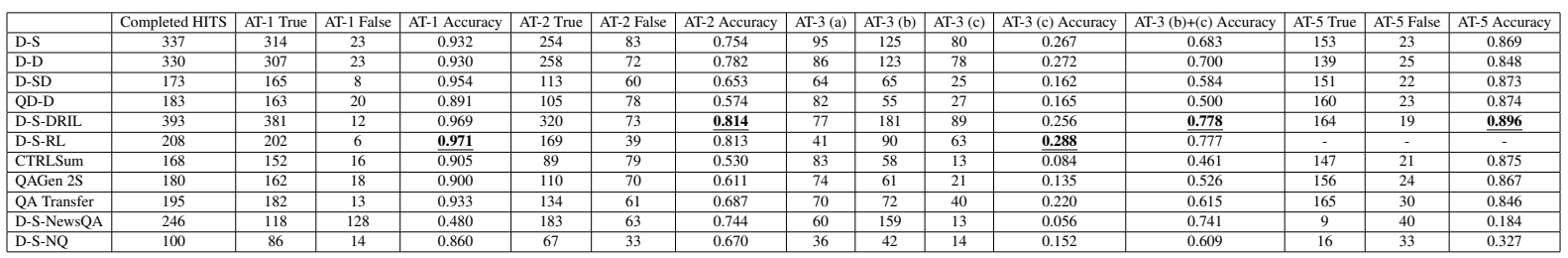

Table 18: Round 1 length bucket 2 human annotation. AT-5 annotation for D-S-RL will be in Round 2.

\begin{tabular}{|l|c|c|c|c|c|c|c|}
\hline & AT-5 True & AT-5 False & AT-5 Accuracy & AT-6 Votes & AT-6 Proportion & AT-7 Votes & AT-7 Proportion \\
\hline D-D & 255 & 111 & 0.697 & 371 & 0.215 & 386 & 0.224 \\
\hline D-S & 281 & 85 & 0.768 & 433 & 0.251 & 424 & 0.246 \\
\hline D-S-DRIL & 298 & 68 & $\mathbf{0 . 8 1 4}$ & 472 & $\mathbf{0 . 2 7 3}$ & 457 & $\mathbf{0 . 2 6 5}$ \\
\hline D-S-RL & 288 & 78 & 0.787 & 450 & 0.261 & 456 & $\underline{\mathbf{0 . 2 6 5}}$ \\
\hline
\end{tabular}

Table 19: Round 2 length bucket 0 human annotation.

\begin{tabular}{|l|c|c|c|c|c|c|c|}
\hline & AT-5 True & AT-5 False & AT-5 Accuracy & AT-6 Votes & AT-6 Proportion & AT-7 Votes & AT-7 Proportion \\
\hline D-D & 273 & 98 & 0.736 & 336 & 0.201 & 349 & 0.210 \\
\hline D-S & 290 & 81 & 0.782 & 417 & 0.250 & 420 & 0.253 \\
\hline D-S-DRIL & 299 & 72 & $\mathbf{0 . 8 0 6}$ & 466 & $\mathbf{0 . 2 8 0}$ & 466 & $\mathbf{0 . 2 8 1}$ \\
\hline D-S-RL & 289 & 82 & 0.779 & 448 & 0.269 & 426 & 0.256 \\
\hline
\end{tabular}

Table 20: Round 2 length bucket 1 human annotation.

\begin{tabular}{|l|c|c|c|c|c|c|c|}
\hline & AT-5 True & AT-5 False & AT-5 Accuracy & AT-6 Votes & AT-6 Proportion & AT-7 Votes & AT-7 Proportion \\
\hline D-D & 287 & 80 & 0.782 & 383 & 0.243 & 383 & 0.246 \\
\hline D-S & 300 & 67 & $\mathbf{0 . 8 1 7}$ & 413 & $\mathbf{0 . 2 6 3}$ & 409 & $\mathbf{0 . 2 6 2}$ \\
\hline D-S-DRIL & 297 & 70 & 0.809 & 401 & 0.255 & 397 & 0.254 \\
\hline D-S-RL & 299 & 68 & 0.815 & 376 & 0.239 & 371 & 0.238 \\
\hline
\end{tabular}

Table 21: Round 2 length bucket 2 human annotation. 


\section{Qualitative Analysis}

\section{D.1 Example 1}

The QA pairs generated by each algorithm for the article in Figure 10 are as follows. D-S-DRIL generates the best QA pair in each length bucket. The questions capture the gist of the article and the answers are accurate in all length buckets. On the other hand, D-S, D-S-RL and QAGen $2 \mathrm{~S}$ ' answer in length bucket 1 do not answer the corresponding why question. QA Transfer and CTRLSum generates unfinished sentences (not well-formed) as it cannot control the brevity of the answers. D-S-NewsQA generates a trivial question in length bucket 0 . D-S-NQ's generated questions in length bucket 0 is way off the gist of the article. D-S-DRIL Length Bucket 0 [Question] Are college athletes' voices getting louder and clearer? [Answer] The voices of thousands of college athletes are being heard louder and clearer than they have in years.

D-S-DRIL Length Bucket 1 [Question] Why are college athletes calling for change? [Answer] The voices of thousands of college athletes are being heard louder and clearer than they have in years. From seemingly small issues of inequality in NCAA Tournament weight rooms to life-and-death issues of police brutality and endemic racism, athletes are increasingly calling for change.

D-S-DRIL Length Bucket 2 [Question] Why are college athletes calling for change? [Answer] The voices of thousands of college athletes are being heard louder and clearer than they have in years. From seemingly small issues of inequality in NCAA Tournament weight rooms to life-and-death issues of police brutality and endemic racism, athletes are increasingly calling for change. They joined the \#MeToo movement against sexual harassment and abuse.

D-S Length Bucket 0 [Question] Are college athletes' voices getting louder and clearer? [Answer] The voices of thousands of college athletes are being heard louder and clearer than they have in years.

D-S Length Bucket 1 [Question] Why are college athletes so politically active? [Answer] The voices of thousands of college athletes are being heard louder and clearer than they have in years. It is the most politically and socially active generation in a half-centure, since the turbulent years of the late 1960s and early 70s.

D-S Length Bucket 2 [Question] Why are college athletes so politically active? [Answer] The voices of thousands of college athletes are being heard louder and clearer than they have in years. It is the most politically and socially active generation in a half-centure, since the turbulent years of the late 1960s and early 70s. From seemingly small issues of inequality in NCAA Tournament weight rooms to life-and-death issues of police brutality and endemic racism, athletes

D-S-RL Length Bucket 0 [Question] Are college athletes ready for the future? [Answer] College athletes are increasingly calling for change, intent on molding what the future should look like for everyone.

D-S-RL Length Bucket 1 [Question] Why are college athletes so politically active? [Answer] The voices of thousands of college athletes are being heard louder and clearer than they have in years. It is the most politically and socially active generation in a half-centure, since the turbulent years of the late 1960s and early 70s.

D-S-RL Length Bucket 2 [Question] Why are college athletes so politically active? [Answer] The voices of thousands of college athletes are being heard louder and clearer than they have in years. It is the most politically and socially active generation in a half-centure, since the turbulent years of the late 1960s and early 70s. From seemingly small issues of inequality in NCAA Tournament weight rooms to life-and-death issues of police brutality and endemic racism, athletes are calling

D-D Length Bucket 0 [Question] What's the latest on college sports news? [Answer] The voices of thousands of college athletes are being heard louder and clearer than they have in years.

D-D Length Bucket 1 [Question] Why are college athletes helping to shape politics? [Answer] The voices of thousands of college athletes are being heard louder and clearer than they have in years. It is the most politically and socially active generation in a half-centure, since the turbulent years of the late 1960s and early 70s.

D-D Length Bucket 2 [Question] Why are college athletes involved in politics? [Answer] The voices of thousands of college athletes are being heard louder and clearer than they have in years. It is the most politically and socially active generation in a half-centure, since the turbulent years of the late 1960s and early 70s. From seemingly small issues of inequality in NCAA Tournament weight 
Article (truncated): The voices of thousands of college athletes are being heard louder and clearer than they have in years and it is the most politically and socially active generation in a half-centure, since the turbulent years of the late 1960s and early 70s. From seemingly small issues of inequality in NCAA Tournament weight rooms to life-and-death issues of police brutality and endemic racism, athletes are increasingly calling for change, intent on molding what the future should look like for everyone. Some of the things that have occurred this past year, its encouraged a lot of us to speak out on things, social justice, and how we feel, said Loyola Chicagos Lucas Williamson, who is working on a film project involving the schools 1963 national title team that broke down racial barriers. The things weve seen, going back to last summer, its been emotional for me, Williamson said, and its given me the confidence to go out there and speak on some things I feel confident about, and some things that I feel are just causes. While the movement gained momentum last summer, when George Floyd and Breonna Taylor died at the hands of police and protests hit Americas streets, the reality is that social unrest has been bubbling out of sight for years. It took Colin Kaepernick taking a knee to bring it to the surface. The NFL quarterbacks polarizing stance against social and racial injustice in 2016 was embraced by other pro athletes, and that in turn encouraged college athletes to take a stand. They joined the \#MeToo movement against sexual harassment and abuse, and began threatening to strike to walk off the field of play unless their demands were heard and met. Protests by more than two dozen Missouri football players against on-campus racism led to he ouster of the president of the university system and the chancellor of its flagship campus. And despite pushback from legislators that threatened to strip funding for scholarships, they found support from athletes on campuses across the country...

Figure 10: Article in test set example 1.

rooms to life-and-death issues of police brutality and endemic racism, athletes

D-SD Length Bucket 0 [Question] Why are college athletes speaking out on social issues? [Answer] The voices of thousands of college athletes are being heard louder and clearer than they have in years.

D-SD Length Bucket 1 [Question] Why are college athletes speaking out on social issues? [Answer] The voices of thousands of college athletes are being heard louder and clearer than they have in years. It is the most politically and socially active generation in a half-centure, since the turbulent years of the late 1960s and early 70s. D-SD Length Bucket 2 [Question] Why are college athletes speaking out on social issues? [Answer] The voices of thousands of college athletes are being heard louder and clearer than they have in years. It is the most politically and socially active generation in a half-centure, since the turbulent years of the late 1960s and early 70 s. From seemingly small issues of inequality in NCAA Tournament weight rooms to life-and-death issues of police brutality and endemic racism, athletes

QD-D Length Bucket 0 [Question] Why are athletes speaking out on social issues? [Answer] The voices of thousands of college athletes are being heard louder and clearer than they have in years.

QD-D Length Bucket 1 [Question] Why are athletes speaking out on social issues? [Answer] The voices of thousands of college athletes are being heard louder and clearer than they have in years. From seemingly small issues of inequality in NCAA Tournament weight rooms to life-and-death issues of police brutality and endemic racism, athletes are calling for change.

QD-D Length Bucket 0 [Question] Why are athletes speaking out on social issues? [Answer] The voices of thousands of college athletes are being heard louder and clearer than they have in years. From seemingly small issues of inequality in NCAA Tournament weight rooms to life-and-death issues of police brutality and endemic racism, athletes are calling for change. The movement gained momentum last summer, when George Floyd and Breonna Taylor died at the hands of

QAGen 2S Length Bucket 0 [Question] Why Are College Athletes Calling for Change? [Answer] The voices of thousands of college athletes are being heard louder and clearer than they have in years.

QAGen 2S Length Bucket 1 [Question] Why Are College Athletes Calling for Change? [Answer] The voices of thousands of college athletes are 
being heard louder and clearer than they have in years. It is the most politically and socially active generation in a half-centure, since the turbulent years of the late 1960s and early 70s.

QAGen 2S Length Bucket 2 [Question] Why Are College Athletes Calling for Change? [Answer] The voices of thousands of college athletes are being heard louder and clearer than they have in years. From seemingly small issues of inequality in NCAA Tournament weight rooms to life-and-death issues of police brutality and endemic racism, athletes are calling for change, intent on molding what the future should look like for everyone.

CTRLSum Length Bucket 0 [Question] Why are athletes speaking out on social issues? [Answer] The voices of college athletes are being heard louder and clearer than they have in years.

CTRLSum Length Bucket 1 [Question] Why are athletes speaking out on social issues? [Answer] The voices of college athletes are being heard louder and clearer than they have in years. A new generation of athletes is more progressive on such issues as race, gender and

CTRLSum Length Bucket 2 [Question] Why are athletes speaking out on social issues? [Answer] The voices of college athletes are being heard louder and clearer than they have in years. A new generation of athletes is more progressive on such issues as race, gender and injustice than previous cohorts at the same age, according to a professor of sociology at Skidmore College

QA Transfer Length Bucket 0 [Question] Why are athletes speaking out on social issues? [Answer] According to Andrew M. Linder, a professor of sociology at Skidmore College, there are two main reasons for this athlete-fueled focus on change: First,

QA Transfer Length Bucket 1 [Question] Why are athletes speaking out on social issues? [Answer] According to Andrew M. Linder, a professor of sociology at Skidmore College, there are two main reasons for this athlete-fueled focus on change: First, younger people in general are more progressive on such issues as race, gender and injustice than previous cohorts at

QA Transfer Length Bucket 2 [Question] Why are athletes speaking out on social issues? [Answer] According to Andrew M. Linder, a professor of sociology at Skidmore College, there are two main reasons for this athlete-fueled focus on change: First, younger people in general are more progressive on such issues as race, gender and injustice than previous cohorts at the same age, and second, they have been emboldened by their athletic heroes.

D-S-NewsQA Length Bucket 0 [Question] What is the name of the college athletes? [Answer] The voices of thousands of college athletes are being heard louder and clearer than they have in years.

D-S-NewsQA Length Bucket 1 [Question] What is the most politically active generation in a half centure? [Answer] The voices of thousands of college athletes are being heard louder and clearer than they have in years. It is the most politically and socially active generation in a half-centure, since the turbulent years of the late 1960s and early 70 s.

D-S-NewsQA Length Bucket 2 [Question] What is the most politically active generation in a half centure? [Answer] The voices of thousands of college athletes are being heard louder and clearer than they have in years. It is the most politically and socially active generation in a half-centure, since the turbulent years of the late 1960s and early 70 s. From seemingly small issues of inequality in NCAA Tournament weight rooms to life-and-death issues of police brutality and endemic racism, athletes

D-S-NQ Length Bucket 0 [Question] Who is speaking on the college football debate? [Answer] The voices of thousands of college athletes are being heard louder and clearer than they have in years.

D-S-NQ Length Bucket 1 [Question] What is the political movement in college football? [Answer] The voices of thousands of college athletes are being heard louder and clearer than they have in years. It is the most politically and socially active generation in a half-centure, since the turbulent years of the late 1960s and early 70s.

D-S-NQ Length Bucket 2 [Question] Why are college athletes involved in the civil rights movement? [Answer] The voices of thousands of college athletes are being heard louder and clearer than they have in years. It is the most politically and socially active generation in a half-centure, since the turbulent years of the late 1960s and early 70 s. From seemingly small issues of inequality in NCAA Tournament weight rooms to life-and-death issues of police brutality and endemic racism, athletes 


\section{D.2 Example 2}

The QA pairs generated by each algorithm for the article in Figure 11 are as follows. The article is regarding the impact of Biden's infrastructure plan on Amtrak. We can see that the questions generated by D-S-DRIL in length bucket 1 capture the gist of the article, but the questions generated by D-S and D-S-RL in length bucket 1 do not capture the gist of the article. This shows the advantage of the DRIL which generates better summaries.

D-S-DRIL Length Bucket 0 [Question] Is Biden's infrastructure plan the right thing to do for America? [Answer] President biden's infrastructure plan is what this nation has been waiting for, Amtrak chief executive William J. Flynn said.

D-S-DRIL Length Bucket 1 [Question] What does President biden's infrastructure plan mean for Amtrak? [Answer] President biden's infrastructure plan is what this nation has been waiting for, Amtrak chief executive William J. Flynn said. The federal funding would help Amtrak along-needed upgrades to tracks, tunnels and bridges in the Northeast.

D-S-DRIL Length Bucket 2 [Question] What does President biden's infrastructure plan mean for Amtrak? [Answer] President biden's infrastructure plan is what this nation has been waiting for, Amtrak chief executive William J. Flynn said. The federal funding would help Amtrak along-needed upgrades to tracks, tunnels and bridges in the Northeast, the nations busiest rail corridor. Amtrak has a $\$ 45.2$ billion backlog of projects that it says are needed to bring its assets to a state of good repair.

D-S Length Bucket 0 [Question] Is biden's infrastructure plan the right thing to do for America? [Answer] President biden's infrastructure plan is what this nation has been waiting for, Amtrak chief executive William J. Flynn said.

D-S Length Bucket 1 [Question] What is President biden's infrastructure plan? [Answer] President biden's infrastructure plan is what this nation has been waiting for, Amtrak chief executive William J. Flynn said. Intercity rail would receive up to a 400 percent boost in funding, according to some estimates.

D-S Length Bucket 2 [Question] What does President biden's infrastructure plan mean for Amtrak? [Answer] President biden's infrastructure plan is what this nation has been waiting for, Amtrak chief executive William J. Flynn said. Intercity rail would receive up to a 400 percent boost in funding, according to some estimates. The federal funding would help Amtrak along-needed upgrades to tracks, tunnels and bridges in the Northeast.

D-S-RL Length Bucket 0 [Question] What is the infrastructure bill? [Answer] The \$2 trillion infrastructure package proposes about $\$ 600$ billion of transportation investments, including $\$ 115$ billion to rebuild bridges and highways.

D-S-RL Length Bucket 1 [Question] What is President biden's infrastructure plan? [Answer] President biden's infrastructure plan is what this nation has been waiting for, Amtrak chief executive William J. Flynn said. The $\$ 2$ trillion infrastructure package proposes about $\$ 600$ billion of transportation investments.

D-S-RL Length Bucket 2 [Question] What is President biden's \$2 trillion infrastructure plan? [Answer] President biden's infrastructure plan is what this nation has been waiting for, Amtrak chief executive William J. Flynn said. The \$2 trillion infrastructure package proposes about $\$ 600$ billion of transportation investments, including $\$ 115$ billion to rebuild bridges and highways, $\$ 85$ billion for transit, $\$ 25$ billion to repair and upgrade airports.

D-D Length Bucket 0 [Question] Is biden's infrastructure plan the answer to America's infrastructure crisis? [Answer] President biden's infrastructure plan is what this nation has been waiting for, Amtrak chief executive William J. Flynn said.

D-D Length Bucket 1 [Question] What would President biden's infrastructure plan mean for Amtrak? [Answer] President biden's infrastructure plan is what this nation has been waiting for, Amtrak chief executive William J. Flynn said. Intercity rail would receive up to a 400 percent boost in funding, according to some estimates.

D-D Length Bucket 2 [Question] What would President biden's infrastructure plan mean for Amtrak? [Answer] President biden's infrastructure plan is what this nation has been waiting for, Amtrak chief executive William J. Flynn said. Intercity rail would receive up to a 400 percent boost in funding, according to some estimates. The federal funding would help Amtrak along-needed upgrades to tracks, tunnels and bridges in the 
Article (truncated): President Biden's infrastructure plan is what this nation has been waiting for, Amtrak chief executive William J. Flynn said, while echoing Biden's push to rebuild and improve the busy Washington-Boston rail corridor. Under the White House plan, intercity rail would receive up to a 400 percent boost in funding, according to some estimates, a transformational investment that could bring major rail expansions and millions more riders. The passenger railroad receives about \$2 billion of federal subsidies annually to cover operations in its national and Northeast networks, as well as other grants and funding for state-sponsored service. The \$2 trillion infrastructure package proposes about $\$ 600$ billion of transportation investments, including $\$ 115$ billion to rebuild bridges and highways, $\$ 85$ billion for transit, \$25 billion to repair and upgrade airports, and \$20 billion for safety initiatives to reduce traffic fatalities. The money, to be spent over eight years, also would address mobility, climate and transportation equity concerns. Amtrak on Wednesday unveiled a plan to provide new intercity rail service to 160 communities and expand service in corridors with heightened demand for rail transportation. The passenger railroad also unveiled a map that highlights 30 possible new routes. The federal funding would help Amtrak along-needed upgrades to tracks, tunnels and bridges in the Northeast, the nations busiest rail corridor. Amtrak has a $\$ 45.2$ billion backlog of projects that it says are needed to bring its assets to a state of good repair in the region. Among those projects is the replacement of the Civil War-era Baltimore and Potomac Tunnel in Baltimore, expected to cost $\$ 4.5$ billion. Other improvements could be achieved by replacing the North River Tunnels, a more than century-old structure that carries about 200,000 daily passenger trips beneath the Hudson River between New Jersey and New York. An $\$ 11.3$ billion plan would double the capacity of existing tunnels, which were damaged by Hurricane Sandy in 2012. Amtrak and other rail services could travel more quickly with the elimination of choke points, additional tracks and other improvements. The passenger railroad has identified about $\$ 18$ billion of available or likely to be available funding for projects in the Northeast in the next five years, including the North River Tunnels project...

Figure 11: Article in test set example 2.

\section{Northeast.}

D-SD Length Bucket 0 [Question] How much federal help is President biden's infrastructure plan for Amtrak? [Answer] President biden's infrastructure plan is what this nation has been waiting for, Amtrak chief executive William J. Flynn said.

D-SD Length Bucket 1 [Question] How much would President biden's infrastructure package benefit Amtrak? [Answer] President biden's infrastructure plan is what this nation has been waiting for, Amtrak chief executive William J. Flynn said. Intercity rail would receive up to a 400 percent boost in funding, according to some estimates.

D-SD Length Bucket 2 [Question] How much would President biden's infrastructure package benefit Amtrak? [Answer] President biden's infrastructure plan is what this nation has been waiting for, Amtrak chief executive William J. Flynn said. Intercity rail would receive up to a 400 percent boost in funding, according to some estimates. The federal funding would help Amtrak along-needed upgrades to tracks, tunnels and bridges in the Northeast.

QD-D Length Bucket 0 [Question] How much would Amtrak get from biden's infrastructure package? [Answer] President biden's infrastructure plan is what this nation has been waiting for, Amtrak chief executive William J. Flynn says.

QD-D Length Bucket 1 [Question] How much would Amtrak get from biden's infrastructure package? [Answer] President biden's infrastructure plan is what this nation has been waiting for, Amtrak chief executive William J. Flynn said. Under the White House plan, intercity rail would receive up to a 400 percent boost in funding.

QD-D Length Bucket 2 [Question] How much would Amtrak get from biden's infrastructure package? [Answer] President biden's infrastructure plan is what this nation has been waiting for, Amtrak chief executive William J. Flynn said. Intercity rail would receive up to a 400 percent boost in funding, according to some estimates. The federal funding would help Amtrak along-needed upgrades to tracks, tunnels and bridges in the Northeast.

QAGen 2S Length Bucket 0 [Question] What 
does President biden's infrastructure plan mean for Amtrak? [Answer] President biden's infrastructure plan is what this nation has been waiting for, Amtrak chief executive William J. Flynn said.

QAGen 2S Length Bucket 1 [Question] What does President biden's infrastructure plan mean for Amtrak? [Answer] President biden's infrastructure plan is what this nation has been waiting for, Amtrak chief executive William J. Flynn said. The passenger railroad receives about $\$ 2$ billion of federal subsidies annually to cover operations in its national and Northeast networks.

QAGen 2S Length Bucket 2 [Question] What does President biden's infrastructure plan mean for Amtrak? [Answer] President biden's infrastructure plan is what this nation has been waiting for, Amtrak chief executive William J. Flynn said. The passenger railroad receives about $\$ 2$ billion of federal subsidies annually to cover operations in its national and Northeast networks. The federal funding would help Amtrak along-needed upgrades to tracks, tunnels and bridges in the Northeast.

CTRLSum Length Bucket 0 [Question] How much would Amtrak get from biden's infrastructure package? [Answer] President biden's infrastructure plan is what this nation has been waiting for, Amtrak chief executive William J. Flynn says.

CTRLSum Length Bucket 1 [Question] How much would Amtrak get from biden's infrastructure package? [Answer] President biden's infrastructure plan is what this nation has been waiting for, Amtrak chief executive William J. Flynn said. The $\$ 2$ trillion infrastructure package proposes about $\$ 600$

CTRLSum Length Bucket 2 [Question] How much would Amtrak get from biden's infrastructure package? [Answer] The $\$ 2$ trillion infrastructure package proposes about $\$ 600$ billion of transportation investments, including $\$ 115$ billion to rebuild bridges and highways, $\$ 85$ billion for transit, $\$ 25$ billion to repair and upgrade airports, and $\$ 20$ billion for safety initiatives. Amtrak has a $\$ 45$

QA Transfer Length Bucket 0 [Question] How much would Amtrak get from biden's infrastructure package? [Answer] The passenger railroad receives about $\$ 2$ billion of federal subsidies annually to cover operations in its national and Northeast networks, as well as other grants and funding for state-sponsored service.

QA Transfer Length Bucket 1 [Question] How much would Amtrak get from biden's infrastructure package? [Answer] The passenger railroad receives about $\$ 2$ billion of federal subsidies annually to cover operations in its national and Northeast networks, as well as other grants and funding for state-sponsored service.

QA Transfer Length Bucket 2 [Question] How much would Amtrak get from biden's infrastructure package? [Answer] Under the White House plan, intercity rail would receive up to a 400 percent boost in funding, according to some estimates, a transformational investment that could bring major rail expansions and millions more riders. The passenger railroad receives about $\$ 2$ billion of federal subsidies annually to cover operations in its national and Northeast networks, as well as other grants and funding for state-sponsored

D-S-NewsQA Length Bucket 0 [Question] What is the name of the Amtrak chief executive? [Answer] President biden's infrastructure plan is what this nation has been waiting for, Amtrak chief executive William J. Flynn said.

D-S-NewsQA Length Bucket 1 [Question] What is the name of the plan? [Answer] President biden's infrastructure plan is what this nation has been waiting for, Amtrak chief executive William J. Flynn said. Intercity rail would receive up to a 400 percent boost in funding, according to some estimates.

D-S-NewsQA Length Bucket 2 [Question] What would help Amtrak along? [Answer] President biden's infrastructure plan is what this nation has been waiting for, Amtrak chief executive William J. Flynn said. Intercity rail would receive up to a 400 percent boost in funding, according to some estimates. The federal funding would help Amtrak along-needed upgrades to tracks, tunnels and bridges in the Northeast.

D-S-NQ Length Bucket 0 [Question] Who is President's plan for the US infrastructure? [Answer] President biden's infrastructure plan is what this nation has been waiting for, Amtrak chief executive William J. Flynn said.

D-S-NQ Length Bucket 1 [Question] Who is the President who wants to build America? [Answer] President biden's infrastructure plan is what this nation has been waiting for, Amtrak chief executive William J. Flynn said. Intercity rail 
would receive up to a 400 percent boost in funding, according to some estimates.

D-S-NQ Length Bucket 2 [Question] How much money would be spent on rail in the White House? [Answer] President biden's infrastructure plan is what this nation has been waiting for, Amtrak chief executive William J. Flynn said. Intercity rail would receive up to a 400 percent boost in funding, according to some estimates. The federal funding would help Amtrak along-needed upgrades to tracks, tunnels and bridges in the Northeast.

\section{D.3 Example 3}

The QA pairs generated by each algorithm for the article in Figure 12 are as follows. The article is about how Argentina dealt with the second wave of the COVID-19 infections. We can see that the QA pairs generated by D-S-DRIL in all length buckets are good-the questions are self-contained and summary-centric while the answers are correct. Questions generated by QAGen $2 S$ in length bucket 0 and 1 do not have accurate answers. Questions generated by QD-D, D-S-News and D-S-NQ do not capture the gist of the article.

D-S-DRIL Length Bucket 0 [Question] How is Argentina coping with a second wave of coronavirus? [Answer] Argentina facing a second wave of infections, the South American nation has adjusted its strategy to prioritize protecting its fragile economy.

D-S-DRIL Length Bucket 1 [Question] How will Argentina respond to COVID-19 pandemic? [Answer] Argentinas President Alberto Fernandez was clear when COVID-19 first hit the country early last year. Now facing a second wave of infections, the South American nation has adjusted its strategy to prioritize protecting its fragile economy.

D-S-DRIL Length Bucket 2 [Question] How is Argentina coping with the second wave of coronavirus? [Answer] Argentina has adjusted its strategy to prioritize protecting its fragile economy. It is hoping greater experience dealing with the coronavirus, a nascent vaccine program, and short, regional lockdowns can help keep the virus in check. The second wave comes at a delicate time for the center-left Peronist government.

D-S Length Bucket 0 [Question] Can regional lockdowns keep COVID out of Argentina? [Answer] Argentina facing second wave of coronavirus infections. Short, regional lockdowns can help keep virus in check.

D-S Length Bucket 1 [Question] How is Argentina coping with a second wave of coronavirus? [Answer] Argentina facing a second wave of infections, the South American nation has adjusted its strategy to prioritize protecting its fragile economy. It is hoping greater experience dealing with the coronavirus and short, regional lockdowns can help keep the virus in check.

D-S Length Bucket 2 [Question] Can regional lockdowns stop the second wave of coronavirus? [Answer] Argentina facing second wave of coronavirus infections. Government hopes short, regional lockdowns can help keep virus in check. Second wave comes at delicate time for the center-left Peronist government. It is heading for mid-term elections in October to defend its majority in Congress.

D-S-RL Length Bucket 0 [Question] How is Argentina coping with a second wave of coronavirus? [Answer] Argentina facing second wave of coronavirus infections. South American nation has adjusted strategy to prioritize protecting economy. D-S-RL Length Bucket 1 [Question] How is Argentina coping with a second wave of coronavirus? [Answer] Argentina facing a second wave of infections, the South American nation has adjusted its strategy to prioritize protecting its fragile economy. It is hoping greater experience dealing with the coronavirus, a nascent vaccine program, and short, regional lockdowns can help keep the virus in check

D-S-RL Length Bucket 2 [Question] How will the second wave of COVID-19 affect Argentina's economy? [Answer] Argentinas President Alberto Fernandez was clear when COVID-19 first hit the country early last year: saving lives at all costs trumped any economic concerns. Now facing a second wave of infections, the South American nation has adjusted its strategy to prioritize protecting its fragile economy.

D-D Length Bucket 0 [Question] How can regional lockdowns help keep COVID-19 out of Argentina? [Answer] Argentina facing second wave of coronavirus infections. Short, regional lockdowns can help keep virus in check.

D-D Length Bucket 1 [Question] Can Argentina Keep Coronavirus in Check? [Answer] Argentina facing a second wave of infections, the South American nation has adjusted its strategy to 


Article (truncated): Argentinas President Alberto Fernandez was clear when COVID-19 first hit the
country early last year: saving lives at all costs trumped any economic concerns. Now facing a second
wave of infections, the South American nation has adjusted its strategy to prioritize protecting its
fragile economy. It is hoping greater experience dealing with the coronavirus, a nascent vaccine
program, and short, regional lockdowns can help keep the virus in check. The second wave comes at a
delicate time for the center-left Peronist government. It is heading for mid-term elections in October
to defend its majority in Congress, its popularity bruised by a strict, lengthy lockdown last year and
the hard economic hit. The grains producer is also in talks with the International Monetary Fund to
revamp some \$45 billion in loans it cannot pay back and needs to fire up economic growth to bring in
much needed hard currency. And creditors are looking for signs of recovery after a sovereign debt
restructuring last year. The Fernandez administration wants to avoid imposing a blanket lockdown,
instead using data on caseloads to establish short-term localized restrictions, reinforce sanitary
measures, and maintain controls over borders, a government source said. The government also wants
to accelerate a vaccine roll-out delayed by shortage of supply, aiming to have all medical workers
and those at high risk vaccinated before the fast-approaching southern winter. Argentinas economy
contracted around $10 \%$ last year, the third straight year of recession, and Economy Minister Martin
Guzman has said it could not withstand another total shutdown. Poverty levels rose to $42 \%$ in the
second half of last year. The country has recorded around 2.4 million coronavirus cases and over
56,000 deaths, and a second wave is building with recent daily cases at 80\% of the peak and rising,
a Reuters tally of official data shows. On Tuesday, infections reached a daily record. The second
wave and incidence of cases could be even worse when the variants take hold, said Toms Orduna, an
infectious disease specialist who advises the government, referring to the P1 Brazilian variant and
others racing through the region....

Figure 12: Article in test set example 3.

prioritize protecting its fragile economy. It is hoping greater experience dealing with the coronavirus and short, regional lockdowns can help keep the virus in check.

D-D Length Bucket 2 [Question] How will regional lockdowns help Argentina keep COVID-19 in check? [Answer] Argentina facing second wave of coronavirus infections. Government hopes short, regional lockdowns can help keep virus in check. Second wave comes at delicate time for the center-left Peronist government. It is heading for mid-term elections in October to defend its majority in Congress.

D-SD Length Bucket 0 [Question] How will Argentina deal with COVID-19? [Answer] Argentina facing second wave of coronavirus infections. Short, regional lockdowns can help keep virus in check.

D-SD Length Bucket 1 [Question] How will Argentina deal with COVID-19? [Answer] Argentina facing a second wave of infections, the South American nation has adjusted its strategy to prioritize protecting its fragile economy. It is hoping greater experience dealing with the coronavirus and short, regional lockdowns can help keep the virus in check.

D-SD Length Bucket 2 [Question] How will Argentina deal with COVID-19? [Answer] Argentina facing second wave of coronavirus infections. Government hopes short, regional lockdowns can help keep virus in check. Second wave comes at delicate time for the center-left Peronist government. It is heading for mid-term elections in October to defend its majority in Congress.

QD-D Length Bucket 0 [Question] How will Argentina deal with COVID-19? [Answer] Argentina facing second wave of coronavirus infections. Short, regional lockdowns can help keep virus in check.

QD-D Length Bucket 1 [Question] How will Argentina deal with COVID-19? [Answer] Argentina facing second wave of coronavirus infections. South American nation has adjusted strategy to prioritize protecting its fragile economy. It is hoping greater experience dealing with the virus, a nascent vaccine program, and short, regional lockdowns can help keep the virus in check

QD-D Length Bucket 2 [Question] How will 
Argentina deal with COVID-19? [Answer] Argentina facing second wave of coronavirus infections. South American nation has adjusted strategy to prioritize protecting economy. Hopes greater experience dealing with virus, a nascent vaccine program, and short, regional lockdowns can help keep virus in check. Second wave comes at delicate time for the center-left Peronist government.

QAGen 2S Length Bucket 0 [Question] How is Argentina dealing with a second wave of coronavirus? [Answer] Argentinas President Alberto Fernandez was clear when COVID-19 first hit the country early last year: saving lives at all costs trumped any economic concerns.

QAGen 2S Length Bucket 1 [Question] How is Argentina dealing with a second wave of coronavirus? [Answer] Argentinas President Alberto Fernandez was clear when COVID-19 first hit the country early last year: saving lives at all costs trumped any economic concerns. Now facing a second wave of infections

QAGen 2S Length Bucket 2 [Question] How is Argentina dealing with a second wave of coronavirus? [Answer] Argentinas President Alberto Fernandez was clear when COVID-19 first hit the country early last year: saving lives at all costs trumped any economic concerns. Now facing a second wave of infections, the South American nation has adjusted its strategy to prioritize protecting its fragile economy.

CTRLSum Length Bucket 0 [Question] How will Argentina deal with COVID-19? [Answer] Argentina faces second wave of coronavirus infections. Second wave comes

CTRLSum Length Bucket 1 [Question] How will Argentina deal with COVID-19? [Answer] Argentina faces second wave of coronavirus infections. Second wave comes at a delicate time for the center-left Peronist government. Argentina is heading for mid-

CTRLSum Length Bucket 2 [Question] How will Argentina deal with COVID-19? [Answer] Argentina faces second wave of coronavirus infections. Second wave comes at a delicate time for the center-left Peronist government. Argentina is heading for mid-term elections in October to defend its majority in Congress, its popularity bruised by a strict, lengthy lockdown

QA Transfer Length Bucket 0 [Question] How will Argentina deal with COVID-19? [Answer]
The Fernandez administration wants to avoid imposing a blanket lockdown, instead using data on caseloads to establish short-term localized restrictions, reinforce sanitary measures, and maintain controls

QA Transfer Length Bucket 1 [Question] How will Argentina deal with COVID-19? [Answer] The Fernandez administration wants to avoid imposing a blanket lockdown, instead using data on caseloads to establish short-term localized restrictions, reinforce sanitary measures, and maintain controls over borders, a government source said.

QA Transfer Length Bucket 2 [Question] How will Argentina deal with COVID-19? [Answer] The Fernandez administration wants to avoid imposing a blanket lockdown, instead using data on caseloads to establish short-term localized restrictions, reinforce sanitary measures, and maintain controls over borders, a government source said. Now facing a second wave of infections, the South American nation has adjusted its strategy to prioritize protecting its fragile economy.

D-S-NewsQA Length Bucket 0 [Question] What is the name of the virus? [Answer] Argentina facing second wave of coronavirus infections. Short, regional lockdowns can help keep virus in check.

D-S-NewsQA Length Bucket 1 [Question] What does Argentina want to do to protect its economy? [Answer] Argentina facing a second wave of infections, the South American nation has adjusted its strategy to prioritize protecting its fragile economy. It is hoping greater experience dealing with the coronavirus and short, regional lockdowns can help keep the virus in check.

D-S-NewsQA Length Bucket 2 [Question] What is the name of the virus? [Answer] Argentina facing second wave of coronavirus infections. Government hopes short, regional lockdowns can help keep virus in check. Second wave comes at delicate time for the center-left Peronist government. It is heading for mid-term elections in October to defend its majority in Congress.

D-S-NQ Length Bucket 0 [Question] What is the cause of the Ebola virus in Argentina? [Answer] Argentina facing second wave of coronavirus infections. Short, regional lockdowns can help keep virus in check.

D-S-NQ Length Bucket 1 [Question] What country was hit by Ebola in 2014? [Answer] 
Argentina facing a second wave of infections, the South American nation has adjusted its strategy to prioritize protecting its fragile economy. It is hoping greater experience dealing with the coronavirus and short, regional lockdowns can help keep the virus in check.

D-S-NQ Length Bucket 2 [Question] What is the cause of the virus in Argentina? [Answer] Argentina facing second wave of coronavirus infections. Government hopes short, regional lockdowns can help keep virus in check. Second wave comes at delicate time for the center-left Peronist government. It is heading for mid-term elections in October to defend its majority in Congress. 